\title{
Multispectrum analysis of the oxygen A-band
}

Brian J. Drouin ${ }^{\mathrm{a}}$, D. Chris Benner ${ }^{\mathrm{b}}$, Linda R. Brown ${ }^{\mathrm{a}}$, Matthew J. Cich ${ }^{\mathrm{a}}$, Timothy J. Crawford ${ }^{\mathrm{a}}$, V. Malathy Devi ${ }^{\mathrm{b}}$, Alexander Guillaume ${ }^{\mathrm{a}}$, Joseph T. Hodges $^{c}$, Eli J. Mlawerd, David J. Robichaude, Fabiano Oyafuso ${ }^{\text {a }}$, Vivienne H. Payne ${ }^{\mathrm{a}}$, Keeyoon Sunga ${ }^{\mathrm{a}}$, Edward H. Wishnow ${ }^{\mathrm{f}}$, Shanshan $\mathrm{Yu}^{\mathrm{a}}$

${ }^{a}$ Jet Propulsion Laboratory - NASA, California Institute of Technology, 4800, Oak Grove Drive, Pasadena, CA 91109-8099, USA

${ }^{b}$ Department of Physics, College of William and Mary, Williamsburg, VA, USA

${ }^{c}$ Material Measurement Laboratory, National Institute of Standards and Technology, 100 Bureau Drive, Gaithersburg, Maryland 20899, USA

${ }^{d}$ Atmospheric and Environmental Research, Lexington, MA, USA

e National Renewable Energy Laboratory, 15013 Denver West Parkway, Golden, Colorado 80401, USA

${ }^{f}$ University of California Berkeley, Department of Physics and Space Sciences Laboratory, Berkeley, CA 94720, USA

\begin{abstract}
Retrievals of atmospheric composition from near-infrared measurements require measurements of airmass to better than the desired precision of the composition. The oxygen bands are obvious choices to quantify airmass since the mixing ratio of oxygen is fixed over the full range of atmospheric conditions. The OCO-2 mission is currently retrieving carbon dioxide concentration using the oxygen A-band for airmass normalization. The $0.25 \%$ accuracy desired for the carbon dioxide concentration has pushed the required state-ofthe-art for oxygen spectroscopy. To measure $\mathrm{O}_{2}$ A-band cross-sections with such accuracy through the full range of atmospheric pressure requires a sophisticated line-shape model (Rautian or Speed-Dependent Voigt) with line mixing (LM) and collision induced absorption (CIA). Models of each of these phenomena exist, however, this work presents an integrated self-consistent model developed to ensure the best accuracy.

It is also important to consider multiple sources of spectroscopic data for such a study in order to improve the dynamic range of the model and to minimize effects of instrumentation and associated systematic errors. The techniques of Fourier Transform Spectroscopy (FTS) and Cavity Ring-Down Spectroscopy (CRDS) allow complimentary information for such an analy-
\end{abstract}


sis. We utilize multispectrum fitting software to generate a comprehensive new database with improved accuracy based on these datasets. The extensive information will be made available as a multi-dimensional cross-section (ABSCO) table and the parameterization will be offered for inclusion in the HITRANonline database.

Keywords: oxygen, atmospheric absorption, collision-induced absorption, multispectrum fitting, spectral lineshapes

PACS: 33.70.-w, 33.70.Fd, 33.70.Jg, 33.15.-e, 33.15.Mt, 33.20.Ea

\section{Introduction}

The quantification of Earth's carbon cycle is essential to a fundamental understanding of Earth's climate. The concentration of carbon dioxide varies temporally and geographically based on the variety of sources and sinks operating in the eco-system, atmosphere, cryo-sphere and oceans. The OCO-2 mission was built and designed as a precision tool for the inference of surface fluxes at the parts per million (ppm or $\mu \mathrm{mol} / \mathrm{mol}$ ) scale across the globe. The method chosen for global atmospheric measurements utilizes reflected and scattered solar radiation in the near infrared $(0.7-2.2 \mu \mathrm{m})$, which is absorbed by oxygen and carbon dioxide in amounts proportional to the airmass and carbon dioxide concentration, respectively. This light is gathered on orbit, dispersed in a grating spectrometer, and detected at three band-wide intervals, two for carbon dioxide and one for oxygen. These measurements, in concert, enable determination of carbon dioxide concentration along the ray paths through the atmosphere. Due to the relatively high abundance of carbon dioxide (> $400 \mathrm{ppm}$ ) the desired $1 \mathrm{ppm}$ accuracy requires high signal to noise (SNR) measurements combined with a high fidelity retrieval of information from the acquired spectra.

Early in the mission development, atmospheric databases [1, 2, 3] were evaluated and found to lack accurate lineshape information at the level required by the OCO/OCO-2 missions [4]. Initial development of the Atmospheric $\mathrm{CO}_{2}$ Observations from Space (ACOS) algorithm [5, 6, 7, 8], relegated spectroscopic information into the multi-dimensional ABSorption COefficient (ABSCO) table that is accessed at runtime so that complex lineshape routines would not be recomputed for millions of retrievals. Improvements to the ACOS algorithm and the ABSCO table [9] were fostered by generation of new laboratory data and truth testing with the Greenhouse Gases Observing 
SATellite (GOSAT) [10] and the Total Carbon Column Observing Network (TCCON)[11] datasets. Since launch, major advances have been made in the spectroscopy of the relevant spectral regions that aim to reduce remaining retrieval biases and persistent systematic features in the retrieval residuals.

Focused efforts to improve the oxygen A-band database using Fourier Transform [12, 13] and Cavity Ring Down Spectroscopy (CRDS) were published in the years leading up to launch including (1) precise and accurate position measurements for multiple isotopologues $[12,14,15](2)$ precise and accurate intensity measurements $[12,14,16,17,18,19,20]$ (3) lineshape narrowing determinations for air and self collisional interactions [12, 13, 14, 17] (4) estimates for the temperature dependence of the broadening parameters [21] (5) determinations of line-mixing (LM) and collision-induced absorption (CIA) for high pressures [22], as well as atmospheric pressures in the P-branch [23] and (6) estimates of the extent of water broadening [24, 25, 26, 27]. Much of this information was absorbed into the most recent update of HITRAN [28], with the exceptions for the self-shift coefficient [14] and partial LM/CIA information [23]. Additionally, the OCO-2 retrieval algorithm uses the full A-band ( $\mathrm{P}$ and $\mathrm{R}$ branches) and so there has remained a need for information beyond these P-branch-only CRDS investigations.

Small but persistent biases between the remotely sensed airmass and validation measurements [29], especially at long paths near atmospheric pressure, necessitated further reconciliation. Considering the known interdependences between line-mixing and collision-induced absorption at high pressures [30], this multispectrum analysis effort was pushed forward in an attempt to critically evaluate the datasets and provide a self-consistent product (a new ABSCO table) for use in the retrieval algorithm. CRDS data allow longpathlength measurements with absolute intensities, providing both lineshape information as well as LM and CIA, however information on narrowing of the lineshape may be diminished in the saturated line-centers and no temperature dependent CRDS data are yet available. The FTS data provide intermediate paths, full band coverage and consistency across a broad pressure and temperature range. These spectra are all modeled with multispectrum fitting software using first the spectral line database HITRAN2012 [28], and then model values are adjusted and fitted for better agreement with the data. Limitations in the analysis of laboratory data are then identified and systematically treated using well characterized atmospheric datasets such as TCCON data in the A-band region recorded simultaneously with other atmospheric variables such as aerosol loading and precipitable water vapor. 
Section 3 of this paper presents the experimental and atmospheric datasets used in this process, followed in section 4 by the rationale and methodology; the results are tabulated, displayed and impacts on atmospheric retrievals are discussed in section 5 and conclusions are given in the final section 6 .

\section{Data}

\subsection{CRDS data}

Measurements performed using the frequency-stabilized cavity ring-down spectrometer located at the National Institute of Standards and Technology (NIST) in Gaithersburg, MD, have been previously described [15, 17] and only the relevant conditions and pertinent calibrations are reviewed here.

The high reflectivity mirrors provide wavelength dependent absorption pathlengths of $\sim 3.5 \mathrm{~km}$ that are precisely determined by measurements of the cavity finesse. The spectrum frequency axis is linked to the free-spectralrange (FSR) of the ring-down cavity which provides an extremely linear and precise frequency that can be accurately calibrated in terms of an external frequency reference, in this case a co-aligned potassium gas cell. The laser frequency mode selection is performed with a high precision wavemeter that provides $\pm 30 \mathrm{MHz}$ uncertainty, and uncertainties of the cavity mode position $(<0.5 \mathrm{MHz})$ and reference laser $(<0.1 \mathrm{MHz})$ are smaller than the probe laser linewidth ( $2 \mathrm{MHz})$.

For CRDS measurements, the measured ring-down decay rate (i.e. inverse decay time) divided by the speed of light yields the total optical-loss-perunit length. Here, this quantity is modeled as the sum of three wavelengthdependent components: cavity mirror losses (measured under empty-cavity conditions), Rayleigh scattering losses (calculated), and the absorption coefficient of the medium. In the present experiments, depicted in Figure 1 and Table 1, the accuracy of the resulting absorption coefficient was limited not by the accuracy of the gas mixture $\left(20.720(43) \% \mathrm{O}_{2}\right)$, but rather by uncertainties in the mirror losses (caused by drift in baseline etalons) and uncertainties in the mean decay time (caused by the finite signal-to-noise ratio of the ring-down decay signals). At relatively high optical depths, transmission through the ring-down cavity was substantially reduced below the measurement threshold, thus preventing measurements of decay times which were less than approximately $4 \mu \mathrm{s}$. This limited the dynamic range of the CRDS measurements and resulted in truncated profiles in spectral regions 
Figure 1: Cavity Ring Down Spectra included in the multispectrum analysis. Pure and air ('NIST standard') were used. Four spectra at room temperature are plotted below: $0.133 \mathrm{kPa}$ ( 1 Torr) $\mathrm{O}_{2}$ (red trace) and three ('NIST standard') air at $0.133,53.3,133.3 \mathrm{kPa}$ (1,400,1000 Torr) in green, blue and black, respectively. Lower panels indicate residuals from the presented analysis, a few segments of the $53.3 \mathrm{kPa}$ (400 Torr) air spectrum were removed from the analysis due to an unresolved calibration issue.
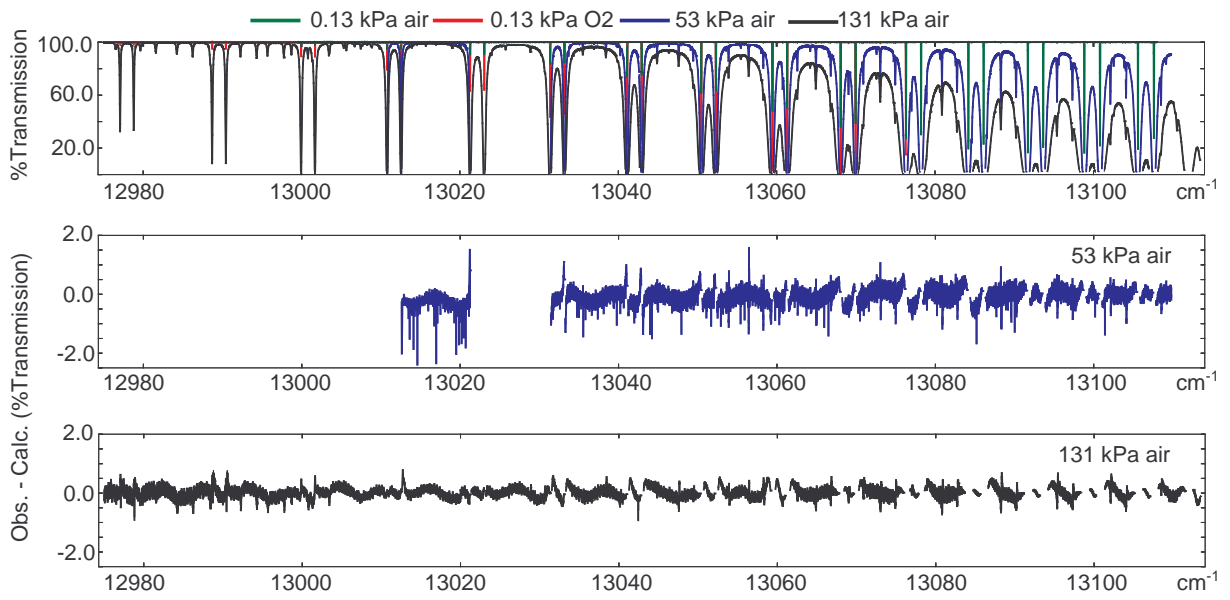

exhibiting strong absorption. The details of the CRDS recordings are given in Table 1.

Reduced spectra were provided for multispectrum fitting for which Rayleigh scattering effects were removed and the cavity loss was normalized to unit path. For introduction into the multispectrum fitting software these spectra were exponentiated and the units correspond to a pathlength of $10 \mathrm{~km}$. Temperatures ranged from 295-298 K and no effort was used to stabilize or control the temperature of the cell. Typically, during the course of a single scan the temperature varied by about $0.1 \mathrm{~K}$. Gas pressure was measured with two NIST-calibrated capacitance diaphragm gauges having full scale responses of $13.3 \mathrm{kPa}$ (100 Torr) and $133 \mathrm{kPa}$ (1000 Torr), respectively, and read-out uncertainties of $<0.01 \%$ and pressures varied about $0.03 \%$ over the course of a scan.

\subsection{Laboratory FTS data}

Several modifications to an absorption gas cell system configured to a Fourier transform spectrometer (FTS), Bruker IFS-125HR, at the Jet Propul- 
Table 1: Datasets and experimental conditions used for multispectrum fitting of the oxygen A-band. Errors (in parenthesis) on pressure, temperature are standard deviations of the measured values, and on pathlength and mixing ratio are from calibration data.

\begin{tabular}{|c|c|c|c|c|c|c|}
\hline Method & $\begin{array}{l}\text { Pres. } \\
(\mathrm{kPa})\end{array}$ & $\begin{array}{c}\text { Temp. } \\
(\mathrm{K})\end{array}$ & $\begin{array}{l}\text { Path } \\
(\mathrm{m})\end{array}$ & $X_{O_{2}}$ & $\begin{array}{c}\mathrm{OPD}^{c} \\
(\mathrm{~m})\end{array}$ & $\begin{array}{r}\mathrm{cal}^{d} \\
\left(\mathrm{x} 10^{10}\right)\end{array}$ \\
\hline CRDS & $0.13112(4)^{a}$ & $295.28(12)$ & $3,500 .^{b}$ & 1.0000 & - & 0 \\
\hline CRDS & $0.1320(1)^{a}$ & $296.31(15)$ & $3,500 .^{b}$ & $0.20720(43)$ & - & 0 \\
\hline CRDS & $53.37(2)$ & $296.55(15)$ & $3,500 .^{b}$ & $0.20720(43)$ & - & 0 \\
\hline CRDS & $131.42(4)$ & $294.65(11)$ & $3,500 .^{b}$ & $0.20720(43)$ & - & 0 \\
\hline FTS & $33.201(33)$ & $297.2(2)$ & 82.73(17) & $0.20720(43)$ & 0.4167 & 186 \\
\hline FTS & $26.771(53)$ & $296.9(2)$ & $99.25(20)$ & $0.20720(43)$ & 0.6429 & 124 \\
\hline FTS & $53.776(107)$ & $297.2(2)$ & $99.25(20)$ & $0.20720(43)$ & 0.4167 & -188 \\
\hline FTS & $40.126(80)$ & $294.9(2)$ & $99.25(20)$ & 0.3250 & 0.4167 & 313 \\
\hline FTS & $19.369(39)$ & $295.8(2)$ & $99.25(20)$ & 0.1057 & 0.500 & 42 \\
\hline FTS & $26.673(16)$ & $297.1(1)$ & $52.00(05)$ & $1.000000(1)$ & 0.375 & -2426 \\
\hline FTS & $67.113(13)$ & $297.5(1)$ & $52.00(05)$ & $1.000000(1)$ & 0.300 & -1606 \\
\hline FTS & $89.502(269)$ & $202.9(14)$ & $52.00(05)$ & $0.20720(43)$ & 0.1125 & -2600 \\
\hline FTS & $109.41(54)$ & $242.6(14)$ & $52.00(05)$ & $0.20720(43)$ & 0.150 & -2600 \\
\hline FTS & $111.26(45)$ & $246.8(12)$ & $52.00(05)$ & $0.20720(43)$ & 0.150 & -2600 \\
\hline FTS & $53.270(53)$ & $209.0(7)$ & $52.00(05)$ & $0.20863(21)$ & 0.225 & -3000 \\
\hline FTS & $133.38(13)$ & $210.2(6)$ & $52.00(05)$ & $0.20863(21)$ & 0.225 & -3000 \\
\hline FTS & 119.91(19) & $187.9(4)$ & $52.00(05)$ & $0.20863(21)$ & 0.225 & -3000 \\
\hline FTS & $56.417(56)$ & $188.3(4)$ & $52.00(05)$ & $0.20863(21)$ & 0.225 & -3000 \\
\hline FTS & $55.310(17)$ & $204.9(6)$ & $52.00(05)$ & $1.000000(1)$ & 0.225 & -3000 \\
\hline FTS & $105.86(21)$ & $206.8(6)$ & $52.00(05)$ & $1.000000(1)$ & 0.225 & -3000 \\
\hline FTS & $96.172(96)$ & $188.7(5)$ & $52.00(05)$ & $1.000000(1)$ & 0.225 & -3000 \\
\hline FTS & $133.28(13)$ & $188.9(4)$ & $52.00(05)$ & $1.000000(1)$ & 0.1286 & -3000 \\
\hline
\end{tabular}


sion Laboratory, were made to enable the collection of high quality oxygen A-band spectra. These modifications include an alternative light source, an external calibration gas cell and use of two different multipass, White-type cells (one operating at ambient room temperature and the other at selected colder temperatures).

To calibrate the wavenumber scales of all the scans, an extra vacuum chamber was configured to the Bruker in order to pass the NIR signal through a sealed $50.8 \mathrm{~cm}$ cell containing about $1 \mathrm{mPa}(7 \mu$ Torr $)$ potassium vapor at room temperature. A source module from an IFS-120HR FTS was configured in this extension chamber for input of NIR radiation through the external source window. An off-axis paraboloid mirror was used to collimate the radiation from a 100 Watt Tungsten filament bulb. A schematic depicting this chamber is given in Figure 2. At the exit of this cell a spherical mirror with focal length $304.8 \mathrm{~mm}$ was used to direct the beam into the FTS. At the resolutions available to this FTS $\left(>0.002 \mathrm{~cm}^{-1}\right)$ the hyperfine and isotopic features of the potassium $\left(D_{1}, D_{2}\right)$ spectrum are important for accurate determination of the wavenumber calibration factor.

Transfer optics placed in the (left) sample compartment of the FTS are used to couple the NIR beam into either of two multipass cells, one a 'Saturn class' White cell purchased from Gemini Scientific Instruments, and the other a custom built coolable White cell originally constructed for far-IR studies at the University of British Columbia (UBC cell [32]) and used recently at JPL to study intensities of the ethane torsional band $\left(\nu_{4}\right)$ near $289 \mathrm{~cm}^{-1}$ [31]. The Gemini cell consists of dielectric silver coated mirrors, optimized for $13000 \mathrm{~cm}^{-1}$ wavenumber region attached to endplates separated by a glass cylinder with vacuum seals. Micrometer adjustments enabled the total path to be adjusted between $82-115 \mathrm{~m}$. The UBC coolable cell was designed for collision-induced absorption studies in the FIR and is capable of holding high density gases ( $<253 \mathrm{kPa}$ or $2.5 \mathrm{~atm})$ at temperatures from 50-300 K. Using $0.05 \mathrm{~mm}$ thick polypropylene windows and gold mirrors the UBC coolable cell provides a maximum $60 \mathrm{~m}$ path.

Details of the UBC cell installed at JPL, and its operation can be found in Ref. [31], however, the temperature control was improved to enable better SNRs for the cold data. Initially, $\mathrm{N}_{2}$ gas was flowed through copper tubing immersed in a dewar of $\mathrm{N}_{2(l)}$ or $\operatorname{Ar}_{(l)}$ to stabilize the flow rate and minimize sample liquefaction. However, better control was achieved for the last set of scans by cooling of the flow gas using a Brooks Polycold gas chiller. A slightly narrower temperature range (176-296 K) compared with [31] was also 
Figure 2: Schematic diagram of external source chamber containing calibration gas cell and 100W Tungsten filament bulb. Optics are standard items from Newport and are aligned with positioning stages prior to evacuation. The rest of the FTS was configured as in Ref. [31]

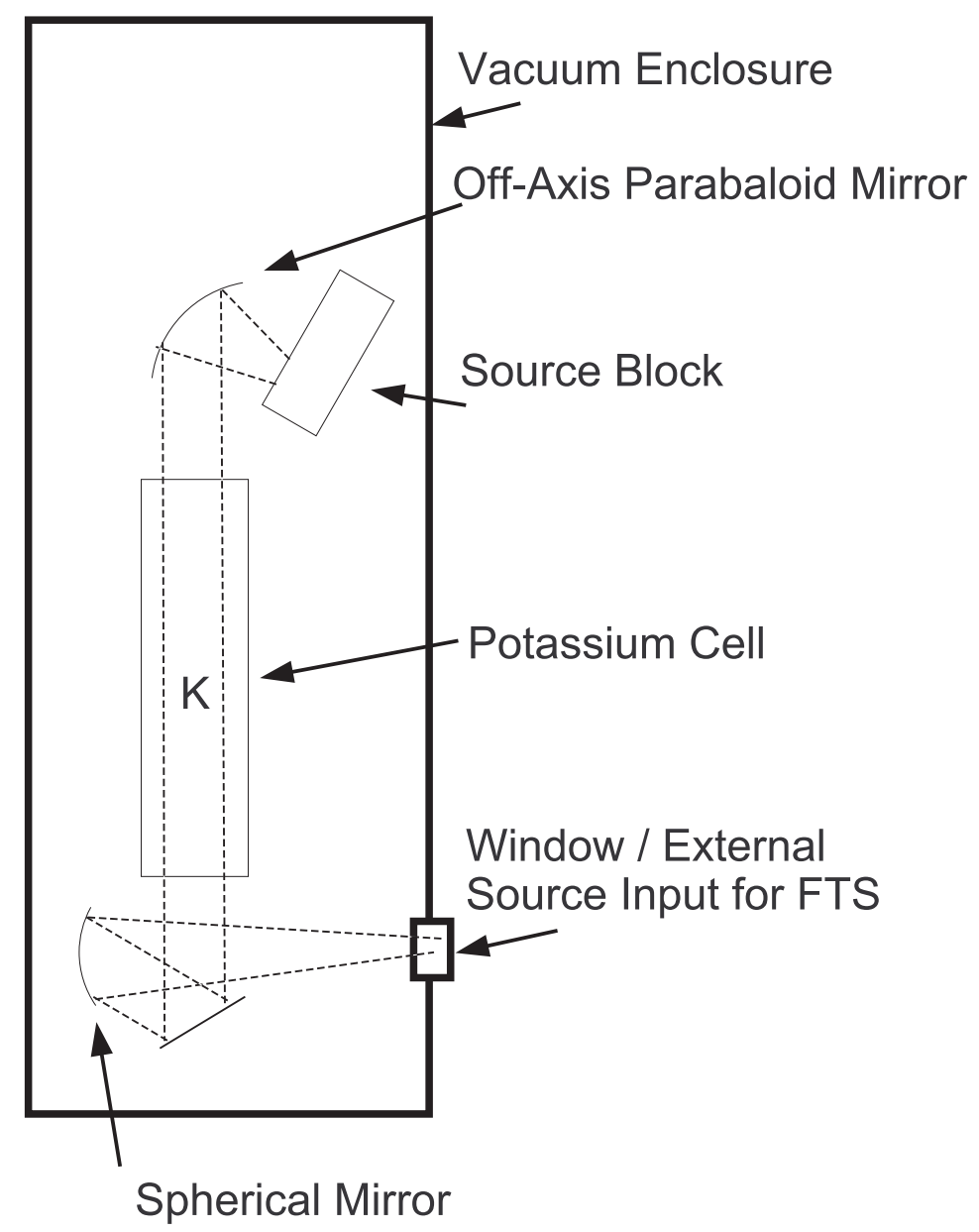


Figure 3: (top) Typical Fourier Transform spectra of air (black) and pure $\mathrm{O}_{2}$ (green) included in the multispectrum analysis. The pathlength is $52.00(5) \mathrm{m}$, the sample pressures are $120 \mathrm{kPa}$ (899 Torr) air and $106 \mathrm{kPa}$ (794 Torr) $\mathrm{O}_{2}$, and the cell temperatures are $187.9(4) \mathrm{K}$ and 206.8(6) K, respectively. (bottom panels) residuals from analysis of the 22 spectra listed in Table 1 described in text.

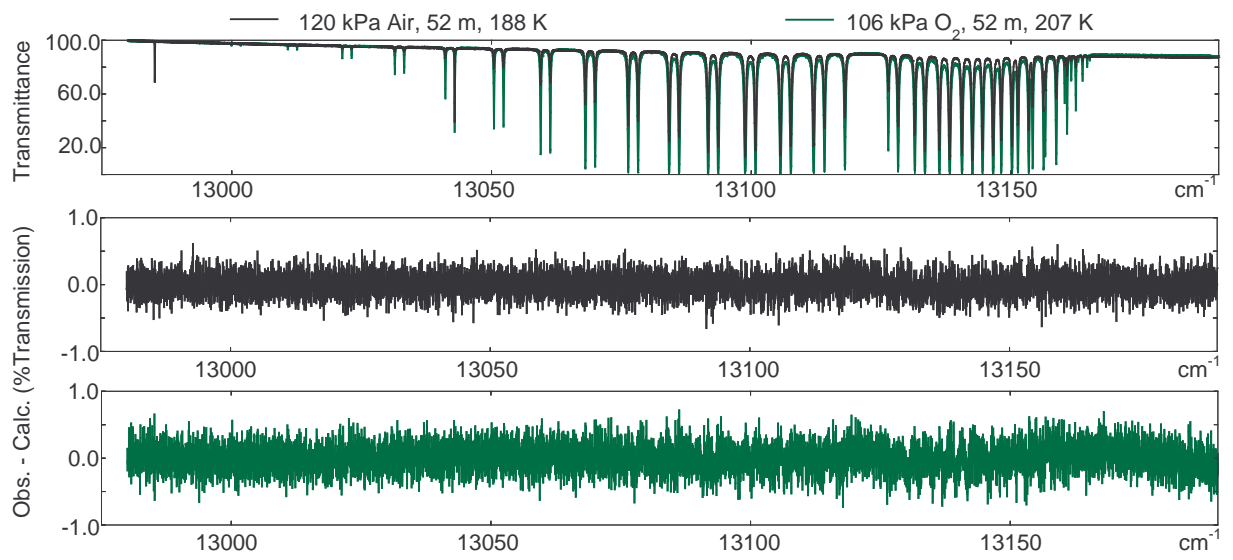

implemented due to the desire to model the Earth atmosphere. The SNR had been limited by use of liquid argon coolant that limited the stability of the cell temperature, which depended critically on 1) the gas flow rate, 2) the number of coils embedded in the liquid argon, and 3) the liquid level itself. Use of the Polycold gas chiller enabled stable temperatures over sufficient recording times to achieve SNRs of $>150$. Two different silicon photo-diodes were utilized for recording of the FTS spectra. Initial data (all of the 'Saturn class' White cell data and several of the UBC cell scans) were recorded with the photo-diode delivered with the spectrometer, however the ability of this detector to pass the fast modulated signals in the NIR was observed to decay over some time, and the apparent zero signal level became unacceptably large. A replacement detector (which came with new preamplifier electronics as well) was found to have improved response time. A listing of the spectra used in the multi-spectrum fit are given in Table 1 and a typical air spectrum is shown in Fig. 3. 


\subsection{Atmospheric FTS data}

TCCON is a network of ground-based FTS instruments that record direct solar spectra in most of the near-infrared, including the OCO-2 spectral bands. The TCCON site in Lamont, Oklahoma, is co-located with the Southern Great Plains (SGP) site of the DoE Atmospheric Radiation Measurement (ARM) program. The SGP facility operates a range of instrumentation, including the Normal Incidence Multifilter Radiometer (NIMFR), used to derive aerosol optical depths from solar radiation at multiple wavelengths.

To provide CIA information across the span of the oxygen A-band directly from well-characterized atmospheric data, a method was developed to analyze the variation of solar direct beam measurements with solar zenith angle for deduction of CIA using atmospheric data. Data selection for this method is critical such that data from periods of time with reasonably stable aerosol and precipitable water vapor (PWV) are utilized while also spanning a range of atmospheric path lengths. To ensure satisfactory conditions, the method combines remote-sensing datasets from the TCCON [11] and NIMFR instruments co-located in Lamont, Oklahoma, as well as ARM data products that specify the temperature and moisture profiles at SGP.

Several criteria were used to select satisfactory time periods on days between January and July 2012 for analysis: (1) the time series of solar irradiance from NIMFR is consistent with clear skies (cloud free); (2) radiance measurements in the OCO-2 bands are reasonably stable; (3) the precipitable water vapor (PWV), as computed by a retrieval from an ARM microwave instrument [33], varies by less than $10 \%$ over a given time period; (4) the aerosol optical depth (AOD) derived from the NIMFR in its $11532 \mathrm{~cm}^{-1}$ channel varies by less than 10\%; and finally (5) the water vapor airmass factor $m_{w}$ has a range (max - min) exceeding 1.5. The $m_{w}$ is taken as defined by Kasten (1966) as:

$$
m_{w}=\left[\cos \theta+0.0548(92.65-\theta)^{-1.452}\right]^{-1}
$$

where $\theta$ is the solar zenith angle in degrees. From the six month interval, 13 cases were selected for analysis and further filtered to eliminate spectra from consideration if its measurement time coincided with an outlier (more than $20 \%$ different than the median for the case) in either the PWV or the AOD measured by the NIMFR.

Both the FTS and NIMFR measure a portion of the solar energy and track the sun, but at differing amounts of collected radiation and with differ- 
ent tracking mechanisms. Comparisons between FTS and NIMFR measurements in a transparent spectral region common to each instrument (11532 $\mathrm{cm}^{-1}$ ) indicated that while the NIMFR data showed smooth temporal behavior, the TCCON FTS data did not. We ascribe this to a solar tracking issue in the FTS. To remedy this issue, a correction procedure that scales an entire TCCON FTS observation, $R(\sigma)$, to match the effective irradiance of temporally matched NIMFR irradiances, was developed. First, a scale factor is obtained for each FTS spectrum by dividing the NIMFR irradiance at $11532 \mathrm{~cm}^{-1}$ by the FTS observation after the NIMFR instrument function for this channel had been applied. This irradiance ratio is then used as a scale factor and applied to corresponding FTS spectra, thereby tying the TCCON data-set to the more stable NIMFR. This scaled irradiance is crucial for binning and sub-sampling the data based on the water vapor airmass factor. For each candidate day of recorded atmospheric spectra, a linear fit to the values of $\ln \left[R\left(11532 \mathrm{~cm}^{-1}\right) / R_{\text {ref }}\left(11532 \mathrm{~cm}^{-1}\right)\right]$ vs. $m_{w}$ is performed. Here $R$ is the FTS measurement and $R_{\text {ref }}$ is a fixed value (such as the greatest value of $R\left(11532 \mathrm{~cm}^{-1}\right)$ for that date). This line is then binned into $m_{w}$ groupings of size 0.5. We then find the value of $R / R_{\text {ref }}$ in each bin closest to the fitted least squares line, and eliminate all other spectra from this case. This vetting process eliminates points that are far from the ideal air mass $\times$ vertical optical depth due to instrumental noise and/or aerosol extinction and gives the data the best possible value of vertical optical depth for subsequent determination of unexplained optical depths. Figure 3.3 provides an example of this procedure for one of the chosen days.

\section{Analysis}

\subsection{Calibrations}

High accuracy spectroscopic methods generally require primary or secondary calibration methods to account for known biases in the measurements. Calibrated pressure gauges (MKS) and platinum resistance thermometers were employed in each experiment, and individual measurements were not limited by the accuracy of these gauges. Shortly after the experiments using the UBC 'cold' cell, a secondary set of gauges was measured with respect to the gauges in this study, then the second set was sent for calibration. The resulting secondary calibration indicates that the primary gauges have maintained the stated accuracy of $0.25 \%$. 
Figure 4: Logarithm of the scaled irradiances (blue dots) (actually $\left.\ln \left[R(11532) / R_{r e f}(11532)\right]\right)$ for July 18, 2012 (1836-2348 UT) plotted vs. water vapor airmass factor, $m_{w}$, and the least-squares fitted line (blue). Also shown (red dots) are the values resulting from the sub-sampling procedure described in the text.

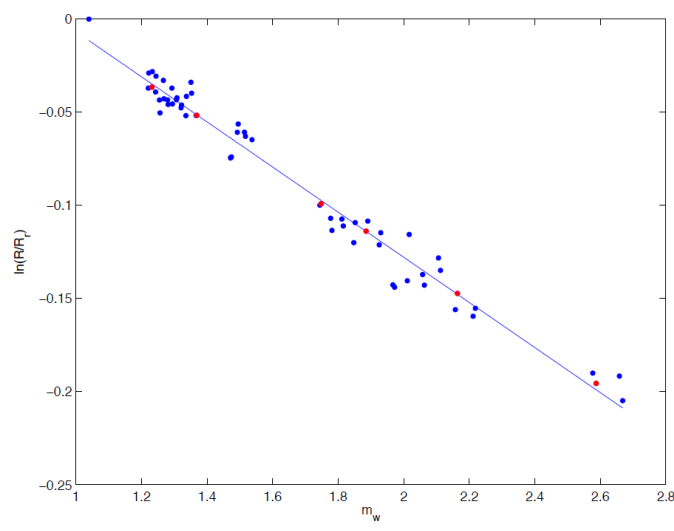


Frequency and intensity calibration of the CRDS data were described in the prior section since calibrated data were provided for this study. For the FTS data, the multispectrum analysis software [34] was used to empirically adjust the measured wavenumbers, $\sigma_{\text {meas }}$, and determine the calibrated wavenumbers, $\sigma_{c a l}$, based on the positions of the potassium $\mathrm{D}_{1}$ (12985.1 $\left.\mathrm{cm}^{-1}\right)$ and $\mathrm{D}_{2}\left(13042.9 \mathrm{~cm}^{-1}\right)$ lines. Although the hyperfine and isotopic line positions of the $D_{1}$ and $D_{2}$ potassium features were well characterized by saturation spectroscopy [35], a complete listing of position and relative intensities was not found in the literature. For the software (described in the following section) it was necessary to create a listing of the calibration gas features in HITRAN format (see Supplemental material). With focus on the $\mathrm{D}_{1}$ and $\mathrm{D}_{2}$ residuals, the potassium gas pressure and the wavenumber scaling factor $d_{1}$ (see Eq. 2) were manually adjusted to minimize these (only these) residuals.

$$
\sigma_{c a l}=\left(1+d_{1}\right) \times \sigma_{\text {meas }}+d_{2}
$$

Values of $d_{1}$ varied from $-4 \times 10^{-8}$ to $+3 \times 10^{-8}$ for the room temperature scans in the Gemini White cell and were all $-26 \times 10^{-8}$ or $-30 \times 10^{-8}$ for the UBC cell, $d_{2}$ was not useful and kept at zero. Variation in the intensity of the potassium absorption was observed since the vapor pressure is strongly temperature dependent, however the low pressure ensures that this variability did not produce any significant pressure shifts. Absolute uncertainties on the calibrated FTS wavenumber scale are about $4 \times 10^{-8}$ relative, or roughly $6 \times 10^{-4} \mathrm{~cm}^{-1}$ absolute $(20 \mathrm{MHz})$.

Some FTS intensity data are not treated explicitly with Beer's law, such that not all contribute to absolute intensity determination. In particular the spectra recorded with the impaired photo-diode required significant (fitted) zero-offsets, $Z$, where transmissions across a given spectrum $T_{i}$ are modified $T_{i}^{\prime}=T_{i}+Z$. Spectra recorded first with the 'Saturn class' White cell required $-0.5 \%--0.9 \%$ offsets, and the three spectra retained from the first set of data with the UBC White cell required $-2.0 \%$ to $-2.3 \%$ offsets. After replacing the detector, fitted values of the zero offset were within the noise level ( $0.5 \%)$ and are essentially zero. Six of these spectra did have the zero-offset fixed at zero, indicating that their transmission depths (like the CRDS data) are considered absolute by the fitting program. 


\subsection{Line mixing model}

The comprehensive study of high-pressure oxygen A-band spectra reported by Tran, Boulet and Hartmann $[22,36]$ included a theoretical derivation of the collisional relaxation matrix based on the infinite-order sudden (IOS) approximation followed by the energy correction sudden (ECS) to the off-diagonal elements [37] as used for $\mathrm{CO}_{2}$ by Niro et al. [38]. Application of the standard angular momentum formulation required the molecule to be approximated as a pure Hund's case $b$, such that a spin-free ECS model, in which the collision rates and scaling lengths were fitted to the line-broadening values, was given. The IOS/ECS formalism allowed for adjustment of the scaling length's for both $\mathrm{O}_{2}-\mathrm{O}_{2}$ and $\mathrm{O}_{2}-\mathrm{N}_{2}$ collisions. The results of the fitting at several temperatures enabled modeling of the off-diagonal elements with a power-law expression. The full matrix of values computed for the air mixing ratio $\left(X_{N 2}=0.79, X_{O 2}=0.21\right)$ is available as supplemental material in Ref. [22], and the $\mathrm{O}_{2}-\mathrm{O}_{2}$ relaxation matrix was provided on request. These two matrices were then utilized to (back) calculate the $\mathrm{N}_{2}-\mathrm{O}_{2}$ relaxation matrix using the binary mixture formula.

\subsection{Data model}

The spectroscopic datasets of this study vary widely in pressure and temperature, but the data are focused narrowly on gaseous oxygen absorption (both resonant and non-resonant) in the presence of itself as well as nitrogen gas. In order to provide a self-consistent spectroscopic model capable of high-fidelity reproduction of atmospheric data, we have fitted the data with both a line-by-line (LBL) spectral lineshape model as well as augmented this model with approximate effects of line mixing and collision induced absorption. The multispectrum fitting program contains a number of different spectral lineshape models, as well as options for effective (Rosenkranz [39]) line mixing or full (W-matrix) line mixing.

\subsubsection{LBL parameters}

Positions and intensities of the oxygen A-band transitions are partially fitted within the analysis. Unlike the broadening, shift and narrowing parameters, the line positions and intensities are well defined quantum-mechanically and expressions that allow interpolation/extrapolation as well as information from other analyses can be confidently employed to improve the physical representation of the data within the model. For zero-pressure line position determinations, a simple Taylor series defining the upper state $\left[^{1} \Sigma(\mathrm{v}=0)\right]$ 
energies (Eq. 3) based on the term value, rotation, and centrifugal distortion parameters was provided to the fitting routine via definitions of auxiliary parameters and mathematical operations.

$$
E^{\prime}\left(b^{1} \Sigma_{g}^{+}, J^{\prime}\right)=G_{0}+B_{0} J^{\prime}\left(J^{\prime}+1\right)-D_{0} J^{\prime 2}\left(J^{\prime}+1\right)^{2}+H_{0} J^{\prime 3}\left(J^{\prime}+1\right)^{3}
$$

The definitions of $B_{0}, D_{0}$ and $H_{0}$ as auxiliary parameters enable the fitting program to determine their values from the spectral data during the fit. Lower state energies for all transitions of $\mathrm{O}_{2}$ in the ${ }^{3} \Sigma_{g}^{-}$state are taken from [40] based on the extended analyses presented by [41] and vetted by [42, 43]. Line positions are then determined by differences between upper and lower state energies: $\sigma_{0}\left(J^{\prime}, E^{\prime \prime}\right)=E^{\prime}\left(J^{\prime}\right)-E^{\prime \prime}$. Small, but significant deviations in $E^{\prime \prime}$ at $J>20$ in comparison to HITRAN2012 result in changes to $B_{0}$, $D_{0}$, and $H_{0}$ outside the reported error bars, even though the determined positions agree within a few thousandths of a wavenumber. The change in lower state energies with respect to HITRAN2012 is depicted in Fig. 5. The divergent groups of flat triplets above $N^{\prime \prime}=20$ observed in this plot indicate the difference in the models lies in the rotational expansion and that the spin-splittings of the two parameterizations are in closer agreement, very small deviations at all $N^{\prime \prime}$ are observed due to the truncation of $E^{\prime \prime}$ to four significant figures in the database.

Intensities of transitions between ${ }^{3} \Sigma$ and ${ }^{1} \Sigma$ states were described by Watson [44] and the necessary lower state $B_{0}, \lambda_{0}$ and $\gamma_{0}$ parameters were adopted from [41] for the calculation of the Hönl-London factors $\left(S_{H L}\right)$. The general expression for intensity includes the empirical Herman-Wallis terms $[14,45] c_{1}$ and $c_{2}$ :

$$
I(J, m, T)=G_{e v} \sigma_{0} S_{H L}(J)\left(1+c_{1} m+c_{2} m^{2}\right) e^{-E^{\prime \prime} / k T}
$$

Where $m$ is equal to $-J^{\prime}$ in the P-branch and $J^{\prime}+1$ in the R-branch. The parameter, $G_{e v}=\frac{8 \pi^{3} \mu_{S O}^{2}}{3 h c Q_{e v r s}}$, is independent of the quanta and is a determinable 'band parameter' along with $c_{1}$ and $c_{2}$. The line intensity depends further on the effective spin-orbit coupled magnetic dipole $\left(\mu_{S O}\right)$, the total electronic, vibrational, rotational and spin partition sum $\left(Q_{\text {evrs }}\right)$ as well as fundamental constants $h$ (Planck's constant), $k$ (Boltzmann's constant) and $c$ (speed of light). The upper state dependence of the Boltzmann factor has been neglected. Together these values give the band intensity, $S_{b}(T)$, via summation over all of the transitions. 
Figure 5: Difference between lower state energies of Yu et al. [40] and HITRAN2012 [28], plotted vs. the quantum number $N^{\prime \prime}$ where $J^{\prime \prime}=N^{\prime \prime}+S^{\prime \prime}$.

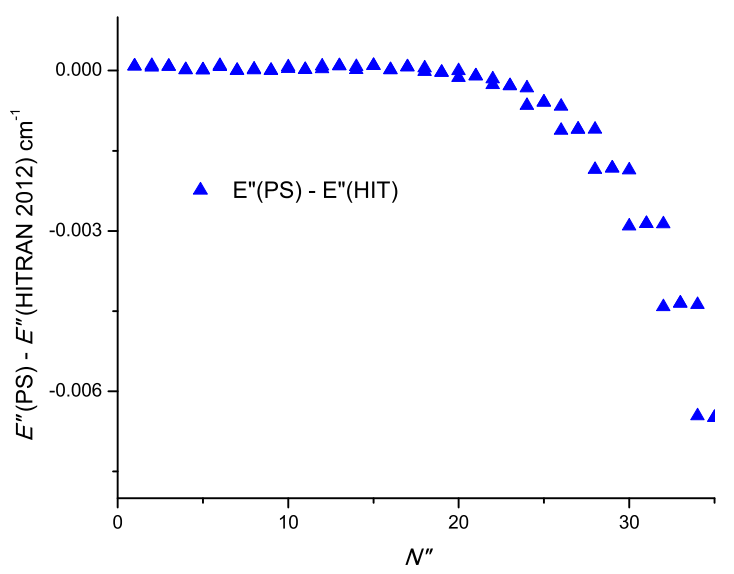

A speed-dependent Rautian lineshape model is chosen for the resonant oxygen lines of the main isotopologue. This formalism incorporates speeddependence as a correction to the Voigt lineshape. The real part (Eq. 5) is used to determine collisional effects inherent to a given transition:

$$
F(x, y, S, H)=\frac{2}{\pi^{3 / 2}} \int_{-\infty}^{+\infty} v e^{-v^{2}} \arctan \left[\frac{x+v}{y\left(1+S\left(v^{2}-\frac{3}{2}\right)\right)+H}\right] d v
$$

in which the frequency detuning relative to the shifted line center $(x)$, as well as the Lorentz width $(y)$ are parameterized for evaluation as an integral through velocity $(v)$ space with the speed-dependence parameter $S$ implicitly included $[46,47]$ and Dicke narrowing $(H)$ optionally included. The variables $x, y$ and $v$ are all dimensionless in this formalism, where $x$ (relative distance to line center) and $y$ (line width) are divided by the Doppler width $\left(\gamma_{D}\right)$ and multiplied by $\left.\sqrt{(} \ln (2)\right)$ for the spectral line and $v$ is divided by the average speed (most probable velocity). This expression omits the farwing effects of the finite collision time described by Ciuryło [47], as well as the speed-dependence of the shift (which would show up in the asymmetric portion described in the following section), but there has been no evidence 
to suggest these effects are significant for the $\mathrm{O}_{2}$ A-band. Ciuryło refers to this expression as the speed-dependent asymmetric Voigt profile (SDAVP), and our omission of negligible terms is similar to other formulations of the speed-dependent Voigt profile [48, 49] and the formulations are expected to be mathematically equivalent. The integral is evaluated numerically over a grid of velocities from $v=-4$ to +4 in sixteen steps, this sampling is sufficient for reproduction of spectra at SNRs of up to 200:1 such as those used in this study. Numerical testing has shown that the approximation fails in different limits for the real and imaginary parts (next section). For the real part the failure occurs far from line center at very long pressure-pathlengths not typically encountered in experimental data. In the fitting algorithm, speeddependence is applied to the total shifted frequency and Lorentz width as calculated from the self and foreign broadened/shifted components. Transitions due to minor isotopologues and potassium transitions that do not require speed dependence are given the simplified Voigt lineshape, equivalent to Eq. 5 with $S=0$. However, a different algorithm is applied for the simple Voigt calculation [50].

Separation of the components of the line shift into pressure and temperature dependent components is given by:

$$
x=\left(\sigma-\sigma_{0}-p\left[X_{f}\left(\delta_{f}+\left(T-T_{r e f}\right) \delta_{f}^{\prime}\right)+X_{s}\left(\delta_{s}+\left(T-T_{r e f}\right) \delta_{s}^{\prime}\right)\right]\right) / \gamma_{D}
$$

where $\sigma$ is the frequency at which the lineshape is to be determined, $\sigma_{0}$ is the center (rest) frequency of the transition; $p$ is the pressure; $X_{f}$ and $X_{s}$ are the mole fractions of the foreign and self broadening gases respectively; $\delta_{f}$ and $\delta_{f}^{\prime}$ are the foreign pressure shift at the reference temperature $T_{r e f}=296 \mathrm{~K}$ and foreign pressure shift temperature dependence, respectively; $\delta_{s}$ and $\delta_{s}^{\prime}$ are the self pressure shift at $T_{\text {ref }}$ and self pressure shift temperature dependence, respectively; and $\gamma_{D}$ is the Doppler width of the transition. Similarly the pressure and temperature dependence of the Lorentz broadening component of the lineshape is given by:

$$
y=\left(p\left[X_{f} \gamma_{f}\left(T_{r e f} / T\right)^{n_{f}}+X_{s} \gamma_{s}\left(T_{r e f} / T\right)^{n_{s}}\right]\right) / \gamma_{D}
$$

where $\gamma_{D}, p, X_{f}$ and $X_{s}$ are as defined in Eq. $6 ; \gamma_{f}$ and $n_{f}$ are the foreign pressure broadening at $T_{\text {ref }}$ and its exponential temperature dependence, respectively; and $\gamma_{s}$ and $n_{s}$ are the self pressure broadening at $T_{r e f}$ and its exponential (power law) temperature dependence, respectively. 
The reference temperature of $296 \mathrm{~K}$ is chosen to make parameters compatible with the HITRAN database, however, the parameters $S, n_{s}, \delta_{s}, \delta_{s}^{\prime}$ and $\delta_{f}^{\prime}$ are not part of the traditional HITRAN parameter set. Fortunately, such parameters can now be incorporated into the recently released HITRANonline database [51], in which extensible formats (such as the Rautian) that are consistent with the Hartmann-Tran profile [52] can be accommodated.

These LBL parameters are defined explicitly for every database entry given in the fitting program. The program computes the contribution of each line to the optical depth of each spectrum and sums the values prior to the transmission calculation as shown in Eq. 8.

$$
\kappa_{i}=\varrho_{O_{2}} \sum_{j=1}^{N} F_{j}\left(x_{i}, y, S\right) I_{j}(J, m, T)
$$

Where the absorption coefficient $\kappa_{i}$ is determined at the $i$ th frequency $(\sigma)$ for all $j$ of the $N$ lines given in the LBL and within a cutoff criterion. The absorption is then converted to transmission using Beer's law; $T_{i}=e^{-L \kappa_{i}}$, using the pathlength, $L$, and oxygen number density, $\varrho_{O_{2}}$, for direct comparison to experimental data. Many of the LBL parameters are not determinable, or correlated with each other and thus inter-dependent, these values may be either fixed to zero or to some reasonable value such as values from the HITRAN2012 database. The lineshape parameters are, in particular, subject to augmentation by the effects of LM, which are accounted for outside the LBL database.

\subsubsection{Line mixing}

In general transitions involving only the same isotopologue can become 'mixed' during the absorption process due to collisional transitions that change the angular momenta of the initial and/or final states. When collisional transition probabilities are low, such that the average energy transfer does not span the distance between transitions, the process is well approximated by line broadening as described by the Lorentzian width given in Eq. 7. However, at high enough pressures where the Lorentzian width becomes comparable to the distance between adjacent transitions the collisional transitions cause line dispersion, with intensity shifting toward the stronger line. Generalized across a band the effect can significantly alter the band-shape at high pressures. 
The amount of line mixing between transition $j$ and transition $k$ can be described using a complex matrix formalism in which the entire relaxation matrix $\mathbf{W}$ represents the fully coupled collisional broadening and shifting across a set of transitions; defined with the complex diagonal elements $W_{k k}=y_{k}+i x_{k}$, and imaginary line-coupling off-diagonal elements, $i W_{j k}=$ $i e^{-E_{j} " / k T} e^{E_{k} " / k T} W_{k j}$, which produce the dispersion at a given pressure and temperature. An implicit transformation of coordinates in Liouville (line) space removes the $\mathbf{W}$ matrix dependence on line positions with the vector $\sigma_{\mathbf{0}}$ removed as $\mathbf{G}(\sigma)=\sigma-\sigma_{0}-i \mathbf{W}$ [53]. The amount of dispersion, $Y_{k}$ of a given line $k$ within the mixed set is dependent only on $\mathbf{G}$, as well as the intensities $I_{j}(T)$, which further depend on state populations at a given temperature, such that the temperature dependence of $\mathbf{W}$ must account for detailed balance. The $\rho$ vector contains the populations, $\rho_{j}(T)=e^{-E_{j} " / k T}$. Intensity information is placed in the vector $\mathbf{X}$ such that $X_{j}=\sqrt{I_{j} / \rho_{j}}$. The full effect of the relaxation matrix on the Lorentz component of the cross section can be written succinctly in matrix form as $Y=\frac{1}{\pi} \operatorname{imag}\left[\mathbf{X}^{\mathbf{T}} \mathbf{G}^{-\mathbf{1}} \rho \mathbf{X}\right]$, however the real portion is already diagonal making full complex matrix manipulation unnecessary. Instead, following [54], the eigenvectors $\mathbf{A}$ obtained from diagonalization of the imaginary part of $\mathbf{G}$ are used to obtain $Y_{k}$ at a given temperature.

$$
Y_{k}(T)=i m a g\left[\sum_{j=1}^{N^{\prime}} \mathbf{X}^{\mathbf{T}} \mathbf{A}+\rho \mathbf{X} \mathbf{A}^{-\mathbf{1}}\right]
$$

provided that the lines within a mixed set of $N^{\prime}$ transitions have their crosssections evaluated together, the entire band intensity is preserved. The dispersive effects of each $Y_{k}(T)$ multiply the imaginary portion of the lineshape:

$$
F^{\prime}(x, y, S, H)=\frac{1}{\pi^{3 / 2}} \int_{-\infty}^{+\infty} v e^{-v^{2}} \ln \left[1+\frac{x+v}{y\left(1+S\left(v^{2}-3 / 2\right)\right)+H}\right] d v
$$

which is calculated along with $F(x, y, S)$ using the LBL parameters. The absorption coefficients are then determined within a mixed set as:

$$
\kappa_{i}=\varrho_{O_{2}} \sum_{k=1}^{N^{\prime}}\left[F_{k}\left(x_{i}, y, S, H\right)+Y_{k}(p, T) F_{k}^{\prime}\left(x_{i}, y, S, H\right)\right] I_{k}(J, m, T)
$$


Remaining transitions outside the mixed set are calculated as before and the total cross section is computed for each spectral element of each spectrum.

An extensive theoretical basis for oxygen LM was published in $[22,36]$ which incorporates an infinite-order sudden approximation of the relaxation matrix. With 66 resonant transitions included in this analysis of the A-band spectrum, the incorporation of theory to determine the $66 \times 66$ elements of $\mathbf{W}$ is essential, especially since the theory indicates that the nearest neighbor approximation (in which a sparse tri-diagonal matrix can be utilized) does not hold for oxygen LM. Rather, the largest off-diagonal elements alternate within the $P$ and $R$ branches, corresponding to strong $J$-coupling within the ${ }^{P} P,{ }^{P} Q,{ }^{R} Q$ and ${ }^{R} R$ sub-branches. Furthermore, spin-couplings between spin-states (i.e. within a ${ }^{P} P-{ }^{P} Q$ or ${ }^{R} Q-{ }^{R} R$ doublet) are also not negligible, and carry the opposite sign compared to the $J$-couplings.

For use of the LM from $[22,36]$ an I/O routine was written that incorporates the lower-triangle of $\mathbf{W}$ into the input file. A schematic representation of the matrix is given in Fig. 6. Since about 5\% uncertainty exists for the accuracy of $\mathbf{W}$, a scaling factor, adjustable by the user at run-time, was incorporated on the off-diagonal elements through definition of auxiliary parameters and constraints in the input file, this also allows for scaling factors due to differing LM formulations. A test output from within the LM subroutine confirmed that the detailed balance calculation reproduces the upper-triangle of $\mathbf{W}$ and that a sign change for the $\mathbf{W}$ matrix is necessary to account for a differing definition of the imaginary portion of the relaxation matrix and the derived Rosenkranz parameters. The sign change is consistent with the sign change in the 're-normalization' process [38] applied in Tran's code that is provided along with the $\mathbf{W}$ matrix, the factor of $\frac{1}{2}$ is a difference in empirical scaling that will be described in more detail later. Further reconciliation of the theoretical LM with the experimental and atmospheric data necessitated the parsing of the theoretical LM into sub-branches in which spin-couplings are optionally removed. The sub-band matrices allow connections of all even $\Delta J$ values.

For this oxygen band, at the sensitivity for which LM becomes relevant, CIA also becomes important [30], so an integrated approach is important to retrieval of self-consistent LBL, LM and CIA.

\subsubsection{Collision Induced Absorption}

The nature of CIA in the oxygen molecule has been debated for almost a century ([55]), and the most detailed work on oxygen has focused on optical 
Figure 6: Schematic representation of the line mixing collisional relaxation $\mathbf{W}$ matrix. Rows and columns each represent a given transition, which may occur in one of four subbands according to ${ }^{3} \Sigma-{ }^{1} \Sigma$ selection rules, primes indicate a different transition within a given sub-band. Diagonal values (blue) are collisional parameters for a given transition per Eqs. 6 and 7. Off-diagonal values are taken from theory $[22,36]$ and incorporated into the data fitting program. Only the lower triangle (yellow and green) are input since the upper triangle (grey squares) is calculated by detailed balance within the program. Inclusion of all yellow (extra-subband) and green (intra-subband) elements results in substantial fitting residuals, but exclusion of the yellow (extra-subband) elements provides an acceptable solution.

\begin{tabular}{|l|l|l|l|l|l|l|l|l|}
\hline & PP & PQ & $\mathbf{P P}^{\prime}$ & $\mathbf{P Q}^{\prime}$ & $\mathbf{R R}$ & $\mathbf{R Q}$ & $\mathbf{R R}^{\prime}$ & $\mathbf{R Q}^{\prime}$ \\
\hline $\mathbf{P P}$ & & & & & & & & \\
\hline $\mathbf{P Q}$ & & & & & & & & \\
\hline $\mathbf{P P}^{\prime}$ & & & & & & & & \\
\hline $\mathbf{P Q}^{\prime}$ & & & & & & & & \\
\hline RR & & & & & & & & \\
\hline RQ & & & & & & & & \\
\hline RR' & & & & & & & & \\
\hline RQ' $^{\prime}$ & & & & & & & & \\
\hline
\end{tabular}


regions where resonant transitions are not found. However, it is generally accepted that CIA exists as a $<10 \%$ component (at 1 atm or $101.325 \mathrm{kPa}$ ) in regions of resonant absorption as well, especially when the absorption bands are weak, such as the triply forbidden ${ }^{3} \Sigma_{g}^{-} \rightarrow{ }^{1} \Delta_{g}$ band near $7900 \mathrm{~cm}^{-1}$ [56]. In the A-band region, the CIA was estimated by Tran, Boulet and Hartmann [22] to be $1 \%$ of the resonant absorption at 1 atm $(101.325 \mathrm{kPa})$. In that study, very high pressures ( $>10 \mathrm{~atm}$ ) were utilized to determine the CIA as a leftover contribution from the resonant line absorption where LM has been explicitly included. The accuracy was limited to $5 \%$ due to uncertainties in the theory as well as the inherent difficulties of modeling overlapping transitions. Later, Long et al. [23] performed laboratory measurements (building on those used here) and showed that the CIA of Tran, Boulet and Hartmann [22] was underestimated in the strongest part of the $P$-Branch. The present work focuses on the atmospheric pressure range, in which the effects of resonant lines still dominate and LM and CIA manifest at or near the noise level of both the laboratory and atmospheric data.

Due to extensive pathlengths, the CRDS and TCCON data are most sensitive to the CIA. In the $131 \mathrm{kPa}$ air CRDS data, up to $4 \%$ 'unexplained' absorption is observed when incorporating a model without CIA. The 53 $\mathrm{kPa}$ air CRDS data also contains up to $1 \%$ 'unexplained' absorption. The presently used CRDS datasets span only the $P$-branch, but the high airmass TCCON data are also sensitive to CIA, and thus we have evaluated improved CIA models using both datasets.

For proper analysis of the LBL and LM parameters using the $53 \mathrm{kPa}$ and $131 \mathrm{kPa}$ CRDS data, it was essential to include an adequate CIA model within the multispectrum fitting program. Since the CIA derived by [22] disagreed with the NIST data across the band, we developed a flexible model based on the resonant line positions to approximate the shape of the "unexplained' absorption in both the CRDS and TCCON data. Recognizing the pseudo-Boltzmann band contours in the feature prompted the use of the entire list of A-band positions and intensities convoluted with a broad Lorentzian to produce a 'CIA' that is similar to the resonant band contour (and completely ad hoc). Although useful in the limited range and sensitivity of the current CRDS data, this CIA model was inadequate for describing the non-resonant absorption in the vicinity of the $R$-branch bandhead and the gap between $P$ and $R$ branches as observed in the atmospheric data set. Nevertheless, the multispectral fitting software was provided with the capability for generic input of CIA in binary pairs (gases X, Y) as either Gaussian or 
Lorentzian features (with a list of positions, widths and intensities) that sum up to give a binary nonresonant absorption, $\alpha(\sigma)_{X Y}$ at a given wavenumber, $\sigma$. This allowed fitting of CIA sensitive data with a reasonable approximation in place during optimization. For this work approximate CIA for pure $\mathrm{O}_{2}$ and $\mathrm{N}_{2}-\mathrm{O}_{2}$ are treated with the partial number densities $\left(\varrho_{X}\right)$ factored in:

$$
\alpha(\sigma)=\varrho_{O_{2}} \varrho_{N_{2}} \alpha(\sigma)_{O_{2} N_{2}}+\varrho_{O_{2}} \varrho_{O_{2}} \alpha(\sigma)_{O_{4}}
$$

Where the $\mathrm{N}_{4}$ contribution is neglected. With this empirical model the CIA at each and every experimental data point is calculated prior to the leastsquares fitting routines, and the effects of the CIA are folded into the transmittance:

$$
T(\sigma)=Z+B(\sigma) e^{-\left[\varrho_{N_{2}} \alpha(\sigma)_{O_{2} N_{2}}+\varrho_{O_{2}} \alpha(\sigma)_{O_{4}}+\kappa(\sigma)\right] \varrho_{2} L}
$$

where the background across a spectrum is represented generally by a Chebyshev polynomial, $B$, and the total transmittance $T$ contains this polynomial as well as the CIA and resonant line information, $Z$ is the zero offset used on some of the FTS spectra and as a piece-wise balancing factor in CRDS spectra that required corrections to approximate CIA models used in the line-by-line parameter fitting. Since the CRDS data are corrected for scattering losses, the transmission profile is background free, and the polynomial, $B(\sigma)$, is unity. Although the effects are minor in the shorter path FTS data, the CIA correction can remove the need for higher order baseline polynomials that would otherwise fit any trace of CIA in these spectra.

With the CRDS data limited to the $P$-branch, the effects of CIA in the $R$ branch are better understood by examination of the 'unexplained' absorption in the TCCON data described in Section 3. For determination of this CIA, the LBL+LM model from this work was used to generate a 'resonant-only' version of the ABSCO table. Using the set of TCCON spectra described in section 3 we then determined the total vertical optical depth as a function of wavenumber from the variation in the measurements with atmospheric path as the solar angle changes. Using the sub-sampled time series of $R(\sigma)$ (the TCCON FTS measurements at wavenumber $\sigma$ ) and their corresponding airmass factors $m_{w}$, the total vertical optical depth is given by the slope of the linear fit of $\log \left[R(\sigma) / R_{\text {ref }}(\sigma)\right]$ vs. $m_{w}$, where $R_{r e f}(\sigma)$ is a fixed value. These vertical optical depths are a starting point for the remaining work, namely the determination of the (as yet) unmodeled absorption due to oxygen collision pairs. The procedure then is to subtract optical depths (ODs) of the known 
Figure 7: Optical depths derived from TCCON FTS spectra (red), sum of $\mathrm{O}_{2} \mathrm{LBL}+\mathrm{LM}$ and LBLRTM optical depths (green), difference of TCCON FTS and $\mathrm{O}_{2}$ LBL+LM and LBLRTM optical depths (orange), aerosol optical depths (cyan) unknown optical depths (blue) and unknown optical depths filtered in $1 \mathrm{~cm}^{-1}$ bins (magenta, see text for description o f filtering method)) for one of the cases analyzed in this study (July $18^{\text {th }}, 2012$, 4/69-2348 UT).

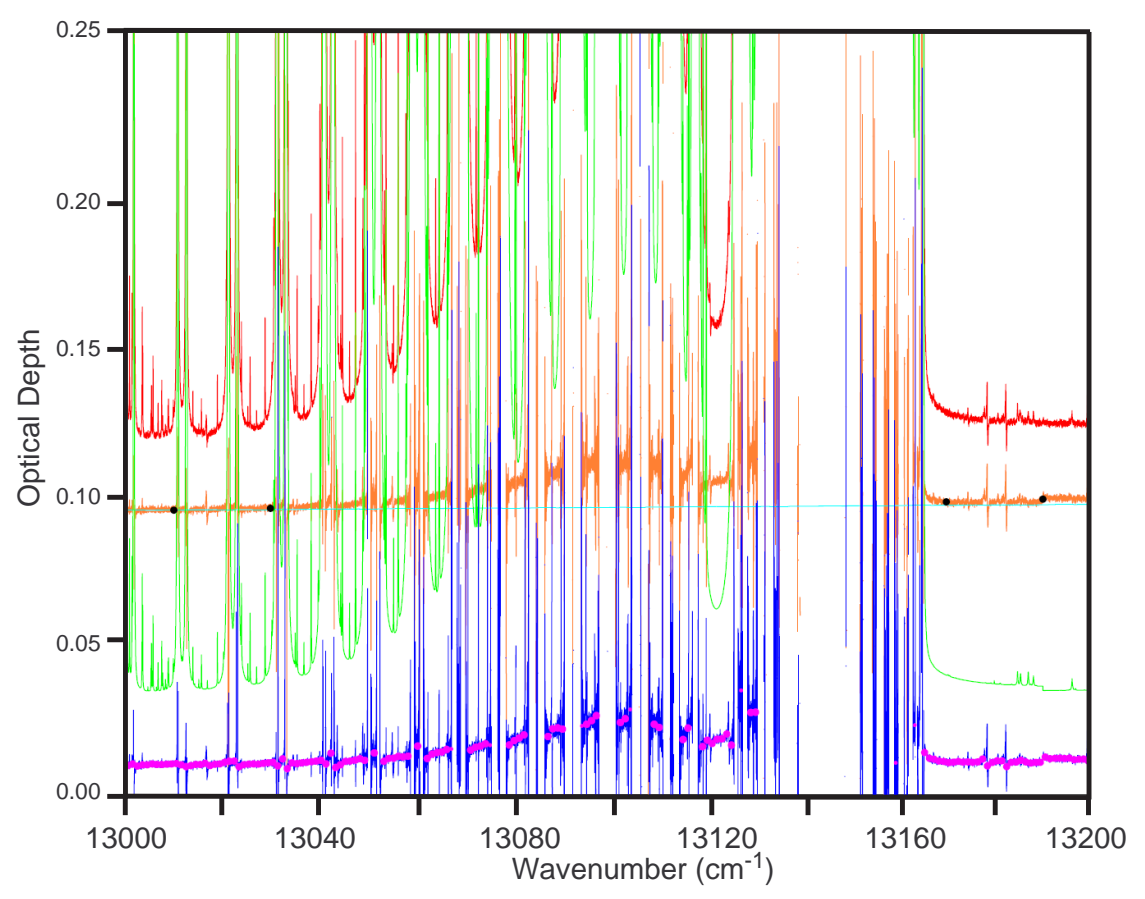

extinction sources from the derived vertical optical depths, thereby obtaining optical depth values from the unmodeled absorption sources.

The calculated ODs, which are the sum of the $\mathrm{O}_{2}$ line ODs from the LBL and LM data analysis described thus far (excluding the empirical CIA) and the ODs from other molecular sources (including Rayleigh scattering) computed by the Line-By-Line Radiative Transfer Model (LBLRTM) [57], are subtracted from the ODs. To remove the contribution of aerosols in the A-band, for each case a spectral Angstrom relation is fitted to this difference at wavenumbers just outside of the $\mathrm{O}_{2}$ A-band $\left.\left(12800 \mathrm{~cm}^{-1}\right)-12950 \mathrm{~cm}^{-1}\right)$, $13250 \mathrm{~cm}^{-1}$ ) - $13400 \mathrm{~cm}^{-1}$ ). (See cyan line in Fig. 7). These spectral aerosol optical depths (AODs) are subtracted from the previously computed 
Figure 8: Comparison with Long et al. 2012 [23] (red) and Spiering et al. 2011 [58](blue) and present study (green) collision induced absorption coefficients for air $\left(\varrho_{O_{2}}=0.21, \varrho_{N_{2}}\right.$ $=0.79)$. One amagat $(\mathrm{am})$ is the density of gas at $101325 \mathrm{~Pa}$ and $273.15 \mathrm{~K}$.

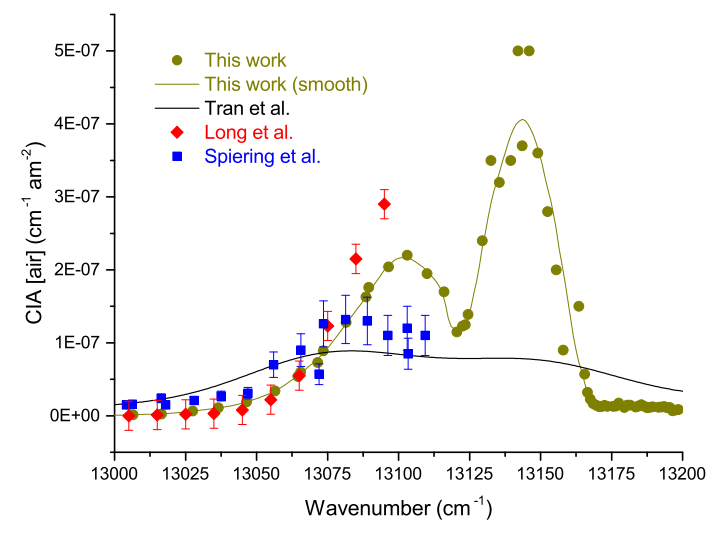

differences, resulting in a spectrum of unexplained optical depths (UODs), which are associated with the A-band CIA.

We then bin the UODs in the $13000-13200 \mathrm{~cm}^{-1}$ region in $1 \mathrm{~cm}^{-1}$ intervals, with roughly 130 spectral points each, and filter the values statistically. Sharp features due to issues with the resonant line (LBL+LM) model are ignored after this filter. Within bins in which 70 or more of the UOD values fall in a narrow range, these comparable values are averaged. Some bins with wider distributions of points are required to have more samples within a wider range. Due to the lower sampling in the latter case, these points (such as the values near $5 \times 10^{-7} \mathrm{~cm}^{-1} / \mathrm{am}^{-2}$ in Figure 8) are weighted less in the determination of a smooth final product. If there are fewer than the looser standard number of points in a bin, there is no representative UOD value for that wavenumber. Fig. 7 shows the elements of the procedure used in the A-band for one case; the magenta points are the representative points resulting from this filtering approach. Figure 8 shows the representative points determined from all 13 representative TCCON data sets that clear the specified cloud, AOD, and PWV conditions. Note the evidence of a possible modeling issue near strong lines, e.g. 13075, 13190, and $13162 \mathrm{~cm}^{-1}$.

We then compute the median value of the UODs for bins with at least three (out of the 13 possible) UOD values. We obtain associated $\mathrm{O}_{2}$-air 
binary absorption coefficient $\left(\alpha, \mathrm{cm}^{-1} /\right.$ amagat $\left.^{2}\right)$ by dividing the representative UODs by the sum of the product of the $\mathrm{O}_{2}$ and air column amounts (in density units). Since the $1 \mathrm{~cm}^{-1}$ binning used to obtain the UOD values is somewhat arbitrary, we manually review each value, resulting in occasional adjustments to both the absorption coefficient and the wavenumber value with which it is associated. Finally, absorption coefficients near strong lines that are likely to have been impacted by the modeling issue mentioned above are removed from the analysis. The final set of absorption coefficients are shown as filled green circles in the Fig. 8. A smooth curve (green curve in Fig. 8) is fitted through these points, providing the final $\mathrm{O}_{2}$ CIA coefficients implemented in the MT_CKD and ABSCO continuum model.

\section{Results and Discussion}

An iterative process, both within and external to the multispectrum fitting, was required for full incorporation of the LBL+LM+CIA model described in the last section. First iterative results from incorporation of LM and CIA resulted in a new self-consistent LBL+LM+CIA model, and then the LBL+LM portion of this model was tested to derive modified CIA from the TCCON data set. Testing of variables revealed that five band parameters, $G_{0}, B_{0}, D_{0}, H_{0}$ (where the subscript indicates the ground vibrational level of the ${ }^{1} \Sigma$ excited state) and $G_{e v}$ were determinable along with LBL parameters for width, shift: $\gamma_{f}, \gamma_{s}, \delta_{f}$ and $\delta_{s}$ for $J$ values $\leq 30$ and LBL parameters for temperature and speed dependence: $n_{f}, n_{s}, \delta_{f}^{\prime}, \delta_{s}^{\prime}$ and $S$ for $J \leq 23$. Some pressure shift parameters for the ${ }^{18} \mathrm{O}^{16} \mathrm{O}$ isotopologue were also floated to minimize residuals further, however it was clear that some issues with the lower state energies and or line positions of these features could not be quickly rectified and these parameters are not reported here.

Initial iterations fitted the CIA effects in the CRDS and FTS data with effective background parameters. The effective backgrounds for the CRDS data could then be used to determine the shape of the CIA as described earlier. The CIA shown in Fig. 8 are the absorption coefficients from (red) [30], (blue)[58], and the (black) values from Tran [22] that are included in HITRAN2012. Our derived values (green) in the A-band P-branch at 13100 $\mathrm{cm}^{-1}$ are in $20 \%$ lower compared to Long et al. [23] and the CIA of Spiering et al.[58] is $50 \%$ of our value at the same wavenumber, however the three data sets converge somewhat at lower wavenumbers and only agree with Tran et al. near $13075 \mathrm{~cm}^{-1}$. Unlike Tran et al. the new CIA in the R- 
branch rapidly drops near the band-head, but does not go to zero. Coupling between these effective backgrounds and the LM was observed and several iterations through the CIA model were performed. The dominant effect of LM was seen in the LBL parameters near the $R$-branch band-head, which showed erratic $m$ dependences. A scale factor of $1 / 2$ was found to dampen the erratic $m$ dependence and significantly reduce curvature between transitions in residuals at high pressures. Unfortunately, this LM also introduced some curvature in the CRDS residuals that could not be compensated with LBL or CIA. Furthermore, comparisons with TCCON data revealed that this augmented LM was inadequate for describing the precipitous drop in absorption at the band head (near $13165.3 \mathrm{~cm}^{-1}$, see Fig. 9). The high pressure FTS data have insufficient SNR to describe the nearly three order of magnitude drop in intensity from the nearest peak (R29Q30), which does not saturate in the laboratory spectra, and is absent from low temperature data. Previous success in this region with the models provided by Tran et al. [22] indicated that our empirical adjustment of LM required better metrics at the band-head. The TCCON data had been well modeled with the ABSCO 4.2 absorption coefficient table which is based on [14], [21], [22] and other information. Therefore, a segment of the ABSCO 4.2 table from $13165-13190 \mathrm{~cm}^{-1}$ at $107 \mathrm{kPa}$ (803 Torr) and $293 \mathrm{~K}$ was placed into the data set as a diagnostic tool. Unfortunately, tests with various LM factors and fits of individual components produced frustrating incompatibilities between this model and in-band LM from FTS (depicted in Fig. 10). The LM matrix provided by Tran, Boulet and Hartmann contains every possible connection between each A-band transition, and it was determined by trial fitting that connections between the ${ }^{R} R$ and ${ }^{R} Q$ sub-bands were introducing residuals into the band-head and band-center regions that were absent when these values were omitted. Omission of all sub-band connections allows one to define the LM with four separate and considerably smaller matrices. Because the intra-subband elements were already the largest values, the remaining portions of the matrix (shown schematically in green in Fig. 6) contain over $90 \%$ of the matrix elements by magnitude, but contain none of the spin-changing terms. The four matrices, one each for ${ }^{P} P,{ }^{P} Q,{ }^{R} R$, and ${ }^{R} Q$ are either $18 \times 18$ for $R R$ and $P P$ or $17 \times 17$ for ${ }^{R} Q$ and ${ }^{P} Q$. By definition these submatrices cannot connect transitions of different spin, which is similar to saying all of the odd $\Delta J$ elements in the $\mathbf{W}$-matrix are negligible. This assumption that spin-changing collisions are negligible is not justified by theoretical arguments about the propensity for spin-changing collisions in 
Table 2: Least-squares fitted values for the $\mathrm{O}_{2}$ A-band ${ }^{1} \Sigma_{g}^{+}(\mathrm{v}=0)$ Hamiltonian. All parameters are given in $\mathrm{cm}^{-1}$.

\begin{tabular}{lcc}
\hline Parameter & This study & Ref. [41] \\
\hline$G_{0}\left(b^{1} \Sigma_{g}^{+}\right)$ & $13122.006033(8)$ & $13122.005748(9)^{a}$ \\
$B_{0}\left(b^{1} \Sigma_{g}^{+}\right)$ & $1.39124765(8)$ & $1.3912493(5)$ \\
$D_{0}\left(b^{1} \Sigma_{g}^{+}\right) \times 10^{6}$ & $5.3631(2)$ & $5.36839(8)$ \\
$H_{0}\left(b^{1} \Sigma_{g}^{+}\right) \times 10^{11}$ & $-0.87(2)$ & $-4.5(\mathrm{f})$ \\
$B_{0}\left(X^{3} \Sigma_{g}^{-}\right)$ & $1.437676036(\mathrm{f})$ & $1.437676036(16)$ \\
$\lambda_{0}\left(X^{3} \Sigma_{g}^{-}\right)$ & $1.984751424(\mathrm{f})$ & $1.984751424(40)$ \\
$\gamma\left(X^{3} \Sigma_{g}^{-}\right) \times 10^{3}$ & $-8.42537576(\mathrm{f})$ & $-8.42537576(290)$ \\
\hline$a$ hypothetical $N=0, J=1$ energy removed
\end{tabular}

relation to rotation-changing collisions [59]. In both the ground state study of [59], as well as Tran et al. [22] models of the odd $\Delta J$ elements are about $10 \%$ of the magnitude of the even $\Delta J$ elements. However, both studies ignored the spin-splitting of the ${ }^{3} \Sigma$ ground state (about $2 \mathrm{~cm}^{-1}$ ), which presumably would further decouple spin states and de-emphasize the importance of odd $\Delta J$ elements. The sub-matrices utilized in the fitting program are provided in the supplemental material.

The even $\Delta J$ elements of the LM, were fixed during optimization of the LBL parameters. Positions and intensities of each transition are defined by the parameters listed in Tables 2 and 3, as well as the lower state energies from Ref. [40]. A comparison of band parameters from this study and the recent work of $\mathrm{Yu}$ et al. [40] is presented in Table 2.

The comparison of line positions in Fig. 11a shows that most positions are systematically shifted from HITRAN2012 by $0.0002 \mathrm{~cm}^{-1}$, a value just above the accuracy of the CRDS measurements and within the calibration accuracy of the FTS data. Since HITRAN2012 is based on average positions from mutliple low pressure CRDS measurements, there are several possible reasons for this small discrepancy (1) inaccurate FTS calibrations are biasing the result; (2) the selected CRDS spectra are on the high end of the mean used for HITRAN2012; (3) pressure shifts were not entirely accounted for in the HITRAN2012 positions. With the discrepancy so near the accuracy of the present data sets, we refrain from conjecture as to the source at this point and maintain that 15 parts per billion knowledge of absolute position is sufficient for remote sensing retrievals. Perhaps the most important aspect of the issue is that the positions and pressure shifts are attained in a self- 
Figure 9: [top left] Selected atmospheric TCCON spectra used to validate results from July $18^{\text {th }}, 2012$ at solar zenith angles of $21^{\circ}$ (brown) and $82^{\circ}$ (blue). [top right] Residuals from prior work (ABSCO 4.2 in red) and present study (PS black) across the A-band, the $21^{\circ}$ solar zenith angle residuals are offset and scaled by the background radiance ratio (40\%) for clarity. Expanded views of residuals in P-branch maximum and R-branch bandhead regions are given at solar zenith angle $21^{\circ}$ [bottom left] and $82^{\circ}$ [bottom right]. The bottom panels depict residuals from prior work (ABSCO 4.2 in red), a rejected analysis incorporating all of the line-mixing parameters from theory (PS (full LM) blue), the analysis presented in this work (PS - black). Spectral radiances are in photons $/ \mathrm{s} / \mathrm{m}^{2} / \mathrm{sr} / \mathrm{cm}^{-1}$, but with arbitrary scaling.
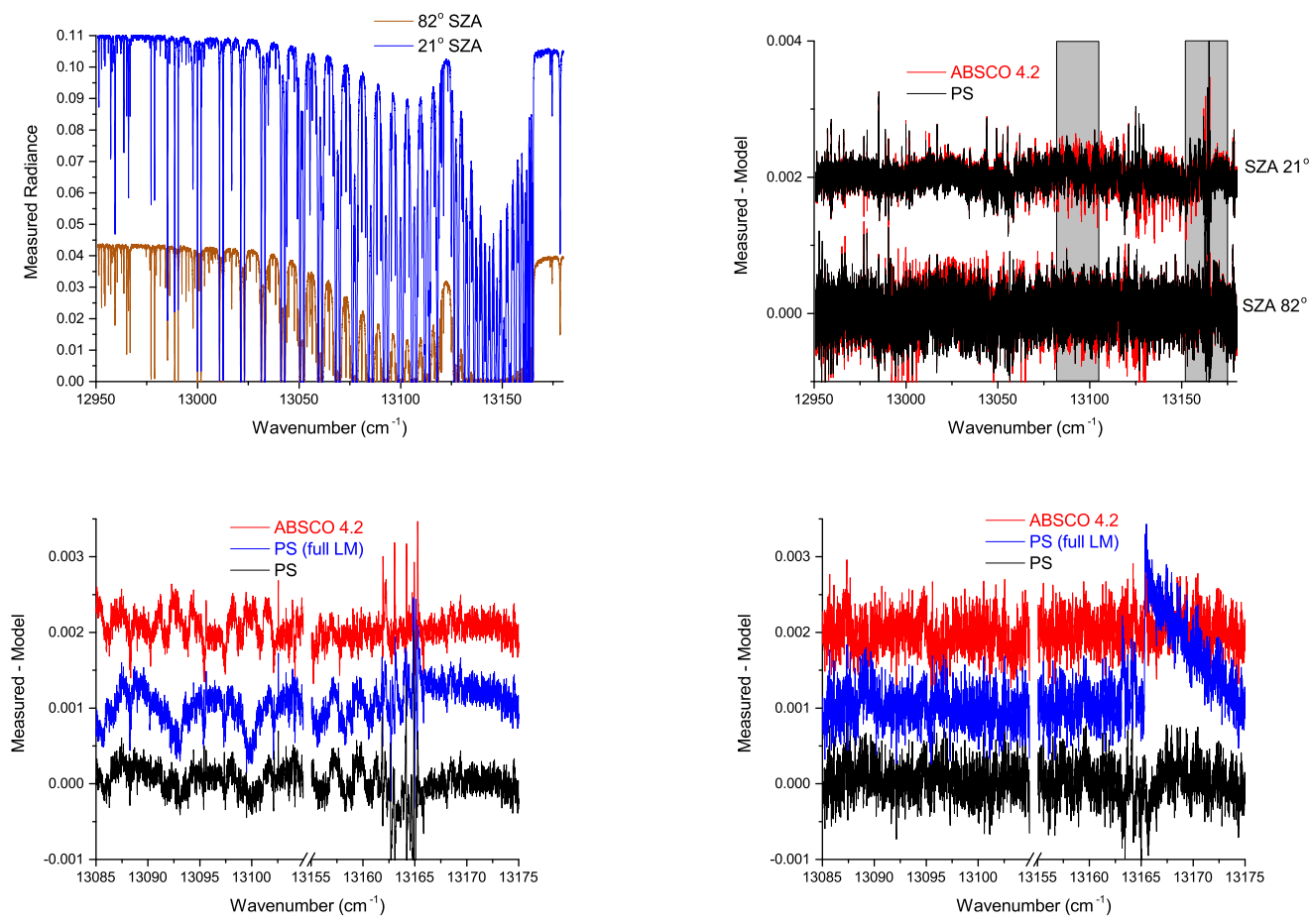
Figure 10: (top) R-branch portion of FTS data scan with pathlength 52.00(5) m, sample pressure $106 \mathrm{kPa}$ (794 Torr) pure $\mathrm{O}_{2}$ and cell temperature $-66.35{ }^{\circ} \mathrm{C}$. (middle) Residuals following LBL optimization with full LM (scaled by 1/2) included. (lower) Residuals following LBL optimization with odd $\Delta J$ LM removed (sub-branch mixing only).
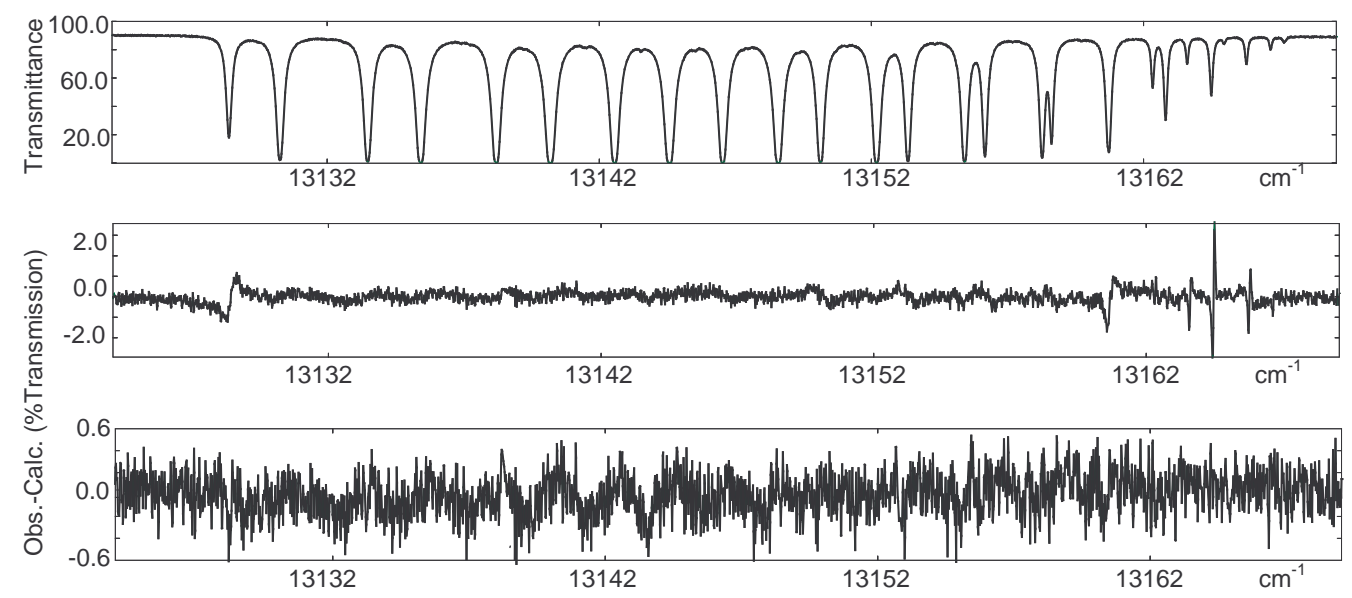

consistent manner from sufficiently accurate data. Further inspection of the line-position differences reveals that $P$-branch values $(m>-30)$ deviate from HITRAN2012 by less than $0.0003 \mathrm{~cm}^{-1}$, still near the accuracy of the data. However, in the $R$-branch a somewhat larger divergence is observed with a $>0.0006 \mathrm{~cm}^{-1}$ difference at $m=30$. This asymmetric behavior was only observed when the lower state energies of $\mathrm{Yu}$ et al. [40] are utilized, whereas fitted positions using lower state energies from HITRAN2012 (not shown) produce a balanced residual pattern, a very large $H$ value and a significantly different $B$ value. Fig. 5 shows that lower state energies from HITRAN2012 deviate from Yu et al. [40] systematically by $>0.001 \mathrm{~cm}^{-1}$ for $|m|>25$, and many common lower state energies disagreed for matching $P$-and $R$-branch transitions in HITRAN2012. It seems that the HITRAN2012 positions were calculated based on fitted $P$-branch values and these mismatched lower state energy values produced a slightly inaccurate prediction of $R$-branch positions above $m=25$. Although the transitions affected are beyond the Boltzmann peak, these are exactly the same transitions that participate strongly in LM near the bandhead and attempts to fit/scale LM prior to fixing the lower state energies to $\mathrm{Yu}$ et al. were largely unsuccessful. The Hamiltonian constraints 
Table 3: Intensity related values for the $\mathrm{O}_{2}$ A-band intensity and ${ }^{3} \Sigma_{g}^{-}(\mathrm{v}=0)$ Hamiltonian.

\begin{tabular}{llll}
\hline Parameter & This study & $\begin{array}{c}\text { Long et al. } \\
{[14] /[60]}\end{array}$ & $\begin{array}{c}\text { Shermaul } \text { et al. } \\
{[61]}\end{array}$ \\
\hline$G_{e v} /(Q a) \times 10^{28}\left(\mathrm{~cm}^{2}\right)$ & $1.1973(9)$ & & $1.217(1)^{a}$ \\
$c_{1} \times 10^{4}$ & $-2.658(\mathrm{f})$ & -2.658 & $5.0(5)$ \\
$c_{2} \times 10^{6}$ & $3.36(\mathrm{f})$ & 3.36 & \\
\hline$\sum I_{k}(296) \times 10^{22}(\mathrm{~cm})$ & $2.2547(2)$ & $2.242(7)$ & $2.270(2)^{b}$ \\
$\mu_{S O}\left(\beta_{e}\right)$ & $0.027430(4)$ & $0.02736(3)^{c}$ & $0.0271^{a}$ \\
${ }^{a}$ factor of $2($ or $\sqrt{2})$ from Hönl-London factor applied \\
${ }^{b}$ present $Q$ gives 2.260 $(2) \times 10^{-22} \mathrm{~cm}$ \\
${ }^{c} 3 j$ normalization factor of $\sqrt{\frac{5}{32}}$ applied
\end{tabular}

combined with consistent lower state energies forces consistency among both upper and lower state energies and does not allow an asymmetric residual associated with (poorly modeled) LM to be adjusted via line position without a consequence elsewhere in the band. The fitted values for $B, D$ and $H$ (see Table 2) were in much better agreement with band parameters reported by $\mathrm{Yu}$ et al. [40] once the lower state energies were corrected to agree with $\mathrm{Yu}$ et al. The precision of $B$ is higher than that given by $\mathrm{Yu}$ et al. [40], and the present value lies about $3 \sigma$ from that value. The presently determined distortion parameter, $D$ is of lower precision, and lies $25 \sigma$ from $\mathrm{Yu}$ et al. [40]. The lack of precision in $D$ and significant deviation from the other study are likely caused by the extra degree of freedom from the present determination of $H$. $H$ was fixed in $\mathrm{Yu}$ et al. [40] based on relations between the Dunham parameters, its value here has the same sign and is about $5 \times$ smaller. Uncertainties listed in Table 4 are precisions and do not reflect potential systematic errors; e.g. line positions uncertainties become as small as $1 \times 10^{-5} \mathrm{~cm}^{-1}(0.3 \mathrm{MHz})$, whereas the frequency calibrations of the FTS data are $6 \times 10^{-4} \mathrm{~cm}^{-1}(20 \mathrm{MHz})$ and frequency measurement in the CRDS dataset has an accuracy closer to $6 \times 10^{-5} \mathrm{~cm}^{-1}(2 \mathrm{MHz})$. The deviation from HITRAN2012, which is based on CRDS data utilized in this analysis, is close to $2 \times 10^{-4} \mathrm{~cm}^{-1}(6 \mathrm{MHz})$ across much of the band.

Figure $11 \mathrm{~b}$ depicts the intensities on a log scale. Relative intensities up to $|m|=35$ from this study in comparison to HITRAN2012 deviate by less than $0.1 \%$, however there is a systematic offset of $0.5 \%$ that results from the implicit inclusion of LM and CIA during the fitting process. It was found that 
the variation of distortion of the transition moment, $c_{1}$ and $c_{2}$ degraded the extrapolated values of the positions and intensities, so these values were held fixed at those determined by [14], who included both CRDS $R$-branch data, as well as high $J$ intensity data from the $P$ branch in their analysis. Fixing these values might indicate that relative intensities would have no standard deviation, however the lower state energies are systematically different and these affect the relative intensities at a fine level. With only one adjusted parameter, the relative intensities and the uncertainties of the intensities are all strictly related by Eq. 4 and a very high absolute precision $\left(<1 \times 10^{-4}\right)$ is attained. There have been many determinations of the intensity of the oxygen $A$ band, and Table 3 outlines a few of the more recently reported values. The table includes determinable band values, as well as integrated band intensities and effective magnetic moments. These are all essentially interchangeable quantities, but have non-trivial interconversions that involve the partition sums, definitions of Hönl-London factors, isotopologue abundance $(a)$, and odd units (1 Debye $=3.33564 \times 10^{-30} \mathrm{C} \mathrm{m}=0.00924$ Bohr Magnetons or $\beta_{e}$ ). Shermaul et al. [61] as well as Brown and Plymate [21] were the first studies to converge at the $1 \%$ level (Brown and Plymate's value is $2.28(2) \times 10^{-22} \mathrm{~cm} /$ molecule) after many prior determinations had discrepancies on the order of $10 \%$. These values remain in close agreement with the (lower) values determined by CRDS $([14,60])$ and FTS+CRDS (this work). The precisions listed in Table 3 are indicative of data quality, but a cursory inspection of the variation from column to column quickly reveals how important accuracy is in these types of measurements. The accuracy of the intensity in our study is actually limited by uncertainty in the various pressure gauges (about $1-2.5 \times 10^{-3}$, or $0.1 \%-0.25 \%$ ), indicating that the determined systematic offset (in comparison to HITRAN2012, based on [14]) of $0.5 \%$ remains statistically relevant.

The LBL parameters listed in Tables 4 and 5 and shown in Figs. 11 and 12 from this study closely resemble values determined by the prior CRDS studies $[14,16]$ which were used to update HITRAN in the HITRAN2012 edition [28]. Plots of the LBL parameters reveal smooth, systematic trends in both the values and the precision uncertainties. A comprehensive comparison to HITRAN2012 was performed as well as inspections of other parameters. 


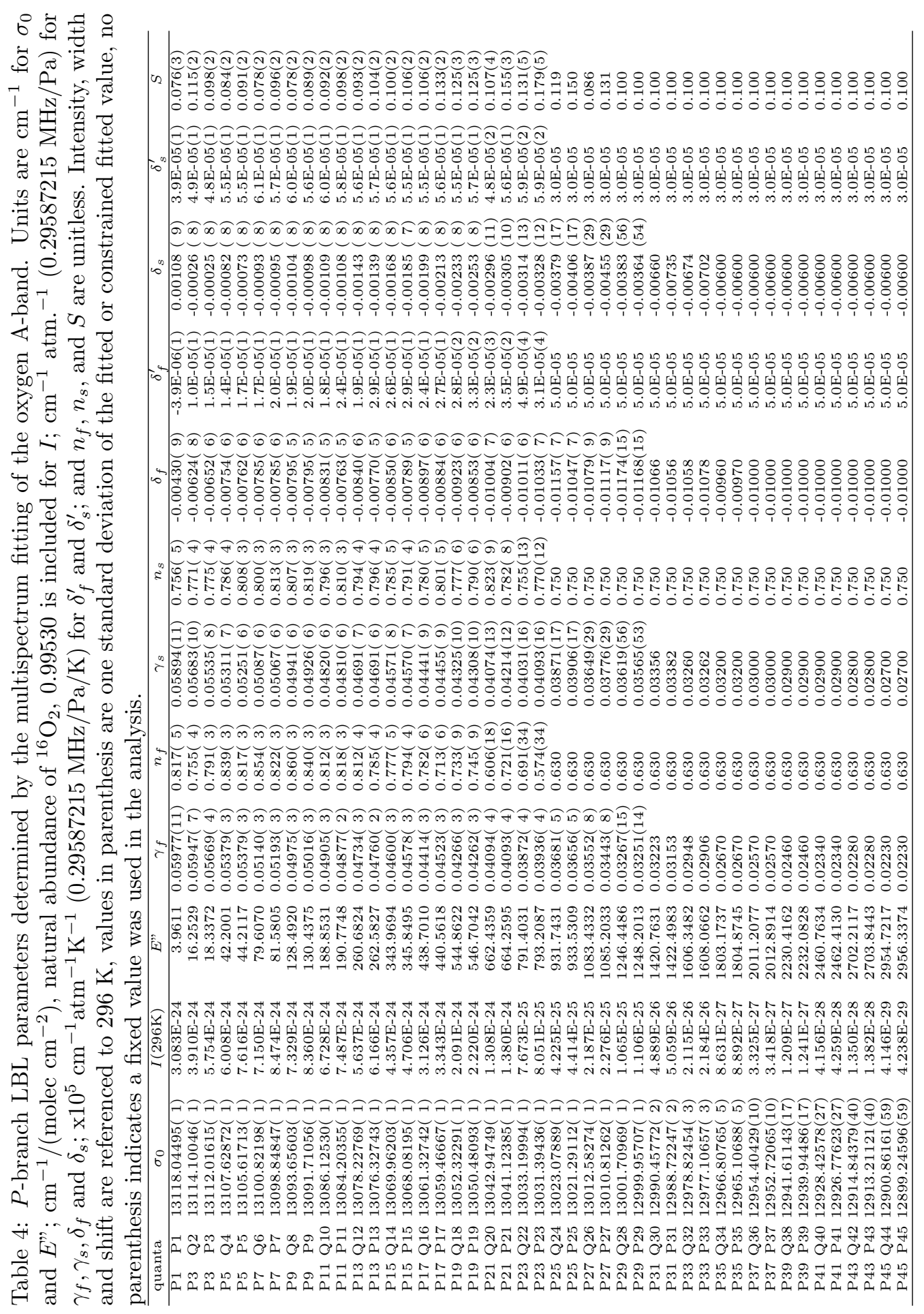


The foreign and self broadening parameters are shown in Figure 11c and $11 \mathrm{~d}$, respectively, units are $\mathrm{cm}^{-1} / \mathrm{atm}(0.29587215 \mathrm{MHz} / \mathrm{Pa})$. The air- and self-broadened widths from HITRAN2012 (as determined by Long et al. [14]) deviate by 3 to $15 \%$. For both broadeners, the deviations at low $J$ occur only for $P 1 P 1, R 1 R 1$ and $P 3 Q 2$ and are roughly $8 \%$ or less. Agreement for the strongest transitions across both $P$ and $R$ is better than $3 \%$ until significant variability is observed in the fitted $R$-branch band head values. However, the variability in the band-head is not as pronounced and with the two outlier widths ( $R 21 Q 22$ and $R 25 R 25)$ are accidentally degenerate lines, producing a strong correlation between their lineshape parameters. The largest deviations all occur where LM is strongest but persist even when LM is set to zero. In the bandhead there is clearly some instability of the solution, and the width values are found to deviate further from the smooth trendline adopted in HITRAN2012 and Long et al. when assumptions about nearby transitions, or fixed values of these transitions are changed. These issues, primarily associated with pressure shifts and temperature dependences of pressure shifts, are discussed further below. Precisions on width values are generally on the order of $0.1 \%$, comparable to the uncertainty due to accuracy of the pressure gauges and do not factor into the determinations of trends and correlations. 
Figure 11: Least squares fitted and fixed values in the LBL for oxygen A-band. (a) position differences (this work - HITRAN2012) [blue], uncertainties in positions [purple], shaded box indicates CRDS accuracy, the FTS accuracy encompasses the chart (b) intensity values at $296 \mathrm{~K}$ [green] and ratios to HITRAN2012 [blue] (c) nitrogen broadened halfwidth coefficients, this work [green], (derived from) HITRAN2012 [blue](d) oxygen broadened halfwidth coefficients, this work [green], HITRAN2012 [blue] (e) nitrogen pressure shift coefficients, this work [green], Long et al.[14] [blue] (f) oxygen pressure shift coefficients, this work [green], Long et al.[14] [blue].

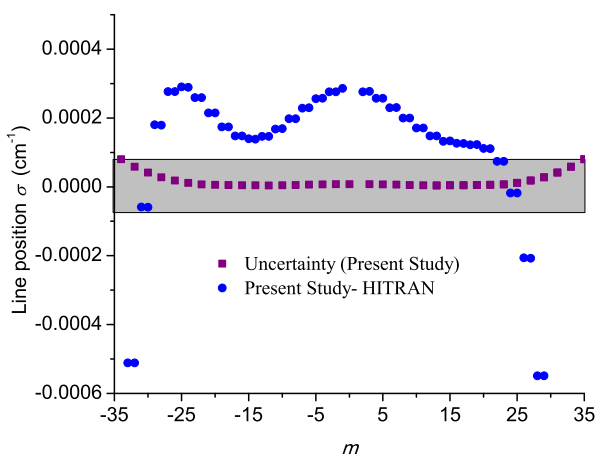

a)

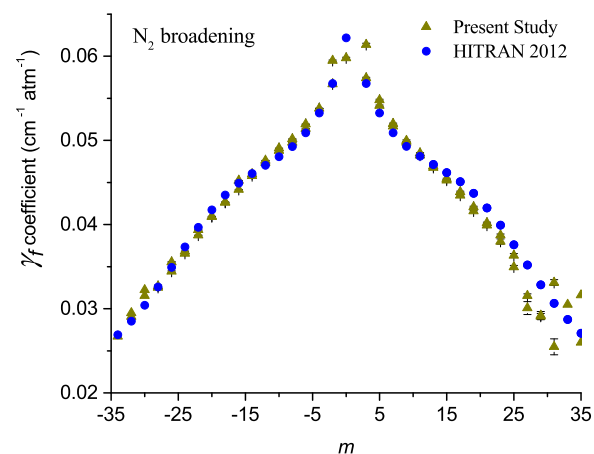

c)

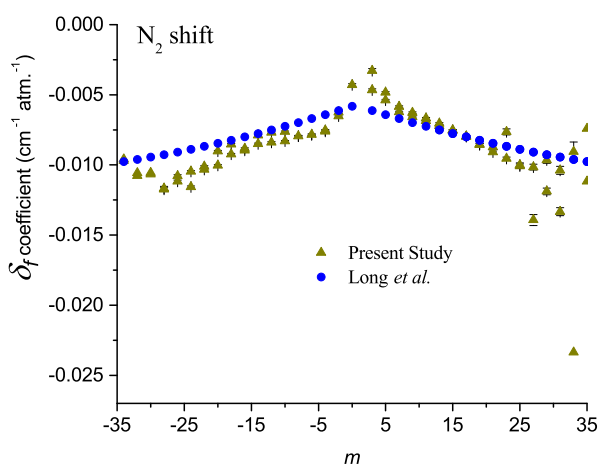

e)

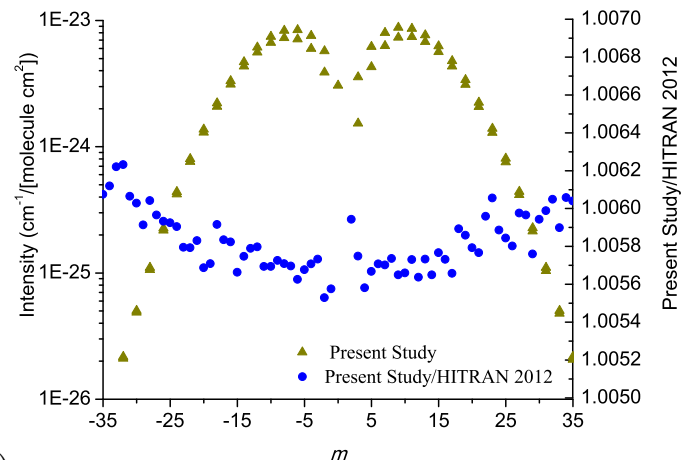

b)

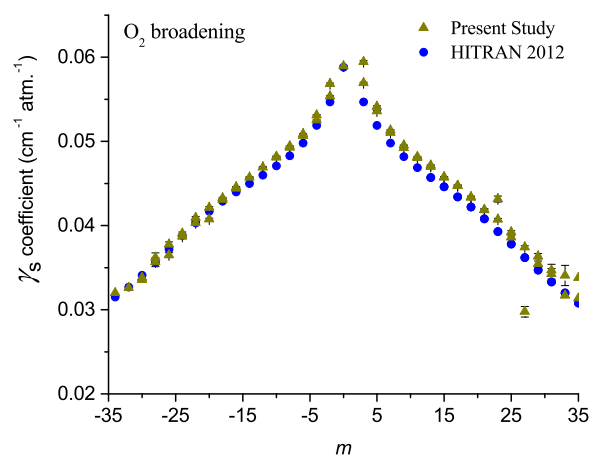

d)

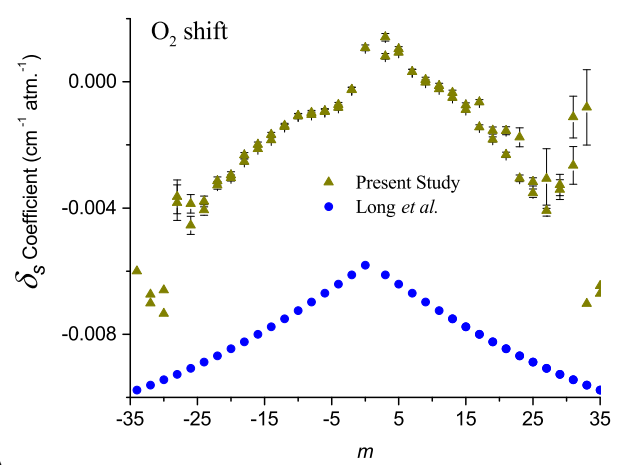

f) 


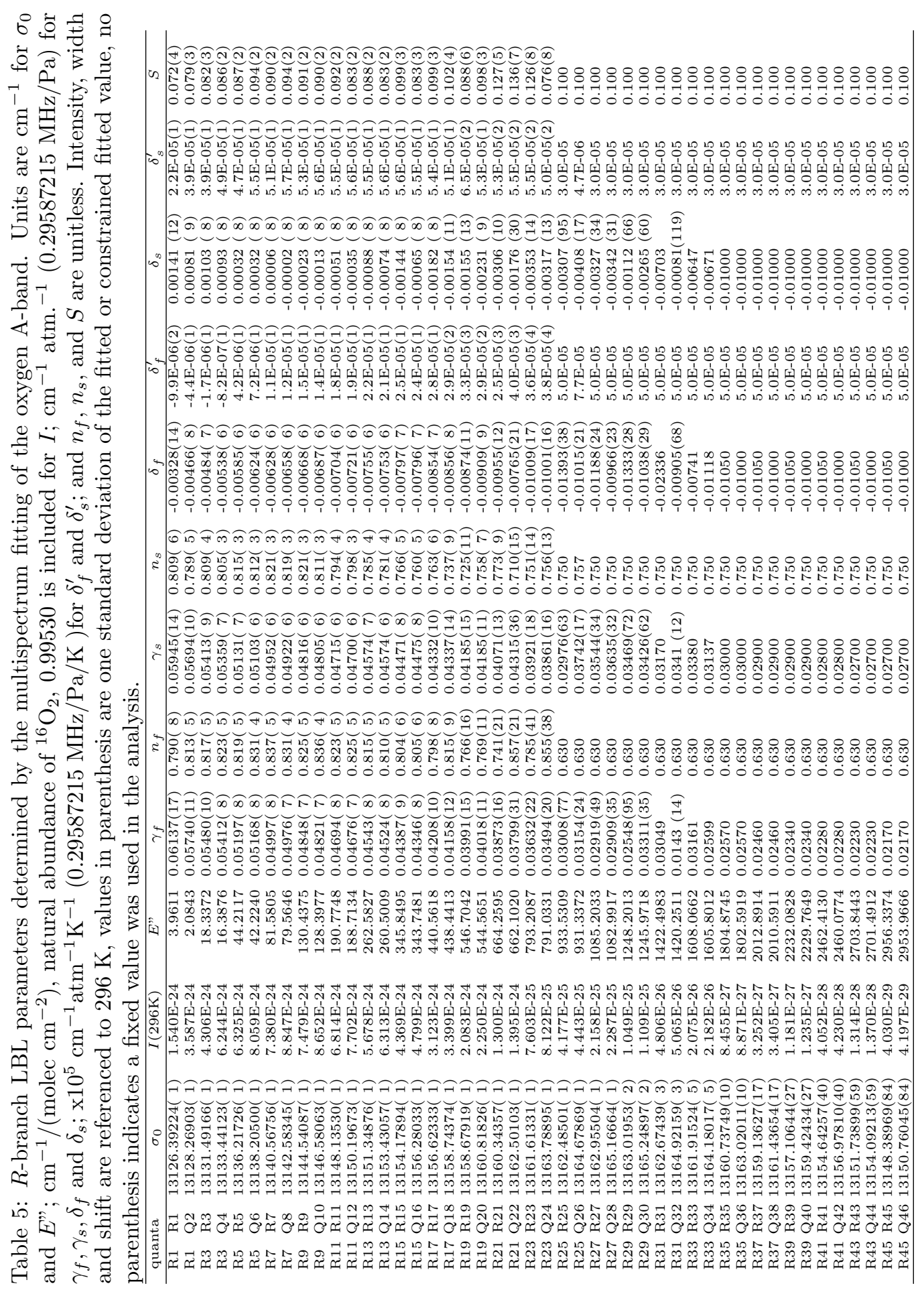


Foreign and self broadened widths have temperature dependences $\left(n_{X}\right)$ that are parameterized with a power law expression in Eq. 7. HITRAN2012 utilized average values from multiple transitions as described by Brown and Plymate [21]. These unitless values are shown in Figures 12a and 12b along with the values determined for each transition in this study. The exponents from Brown and Plymate were deemed to have an accuracy of only $\pm 15 \%$ (error bars in Figures 12a and 12b are based on precision), due to issues with controlling the cell temperature. In contrast, the precisions (error bars) from the present work are under $1 \%$ for most of the band. Based on maximum deviations, the accuracy of the power law exponent is only as good as the accuracy of the width measurement at a representative temperature, divided by the log of the ratio of temperature extrema (see Ref. [49] Eq. 4). The widths are certainly limited by the pressure gauge, which is, at worst, $0.25 \%$. So a measurement range of $200-300 \mathrm{~K}$ gives, at best, $1.25 \%$ accuracy on the exponent. Since the width precisions range from $0.04-1.0 \%$, we give $0.25 \%$ as a lower bound and $1.0 \%$ as an upper bound for the widths and derive a $1.25 \%-5 \%$ range for accuracies on the exponents. This improvement in accuracy allows a statistical comparison to Brown and Plymate, which are, on the average, $8 \%$ smaller (for air), with the strongest lines $(|m|=8)$ being $5,6,12$, and $13 \%$ smaller for nitrogen, nitrogen, oxygen and oxygen; respectively. These strong lines are now estimated to have temperature exponent accuracies of $1.25 \%$, such that a significant distinction between the parameter sets occurs. Sensitivity tests on the OCO-2 algorithm [62] indicate that the change in exponent equates to nearly $1 \mathrm{ppm}$ change in the derived $\mathrm{CO}_{2}$ fraction [62]. Based on the trend up to $J=23$, values of the temperature exponents for $J>23$ were held fixed to 0.63 for $n_{f}$ and 0.75 for $n_{s}$.

The foreign and self pressure shifts $\left(\delta_{X}\right)$ are depicted in 11e and 11f and have units of $\mathrm{cm}^{-1} / \mathrm{atm}(0.29587215 \mathrm{MHz} / \mathrm{Pa})$. HITRAN2012 air pressure shift values are based on the polynomials provided by Long et al. [14], which are equivalent for both oxygen and air shift. Values for this polynomial are plotted along with the foreign pressure and self pressure shifts determined in this study. Values for the nitrogen pressure shift overlap at the strongest transitions, with the current values showing slightly less negative shifts at the center of the band and slightly more negative shifts at the highest quanta fitted, with values near the band head showing significant variability and increased uncertainties. Values for the oxygen pressure shift follow the same trend as for nitrogen, but are all offset to less negative values by $4 \times 10^{-3}$ $\mathrm{cm}^{-1} / \mathrm{atm}$. 
Figure 12: Least squares fitted and fixed values in the LBL for oxygen A-band. (a) nitrogen broadening temperature exponents, this work [green], Brown and Plymate [21] [blue] (b) oxygen broadening temperature exponents [green], Brown and Plymate [21] [blue] (c) linear temperature dependences of the nitrogen pressure shift (d) linear temperature dependences of the oxygen pressure shift (e) speed dependence values (f) dispersion coefficients for air broadening compared with values obtained from Ref. [22] and calculated from data in Refs. [12, 13].

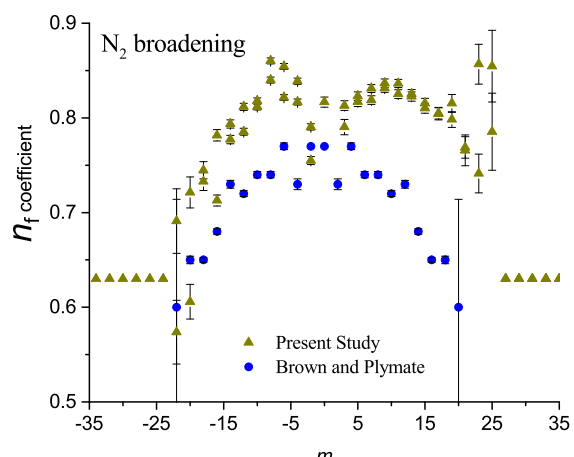

a)

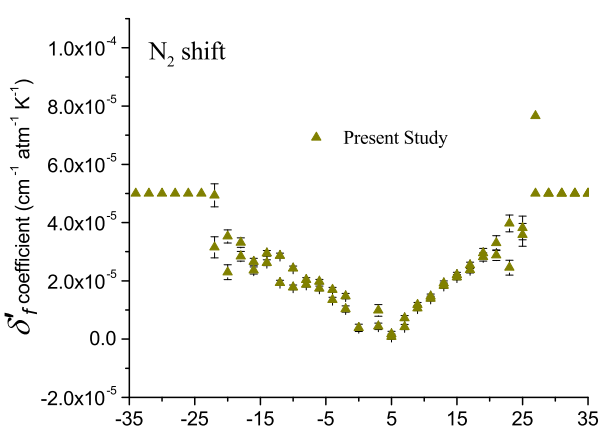

c)

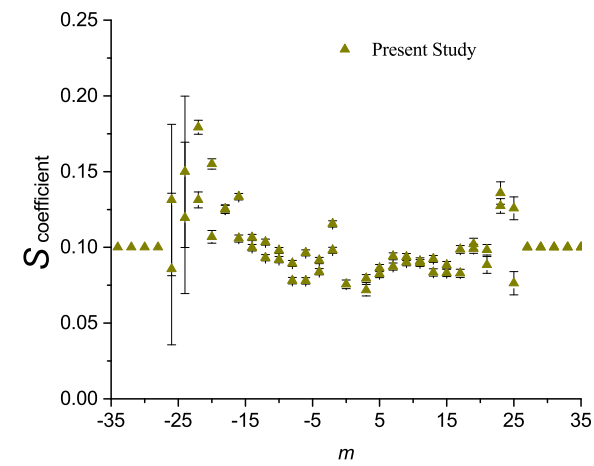

e)

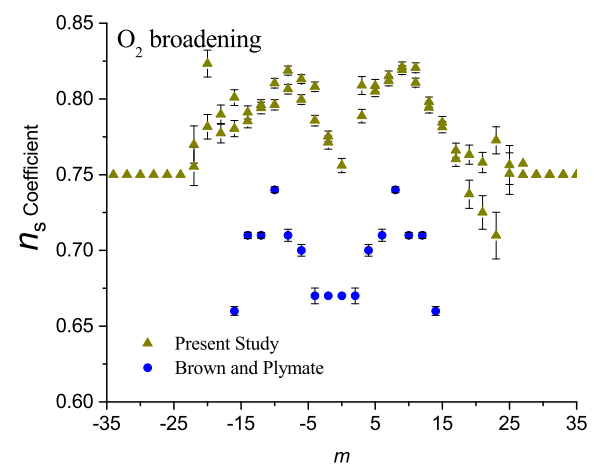

b)

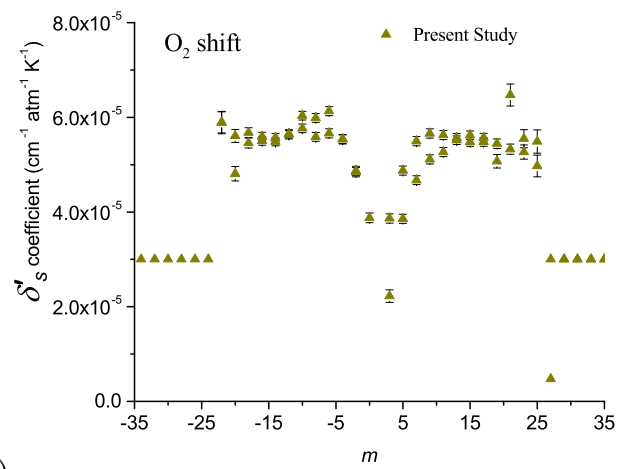

d)

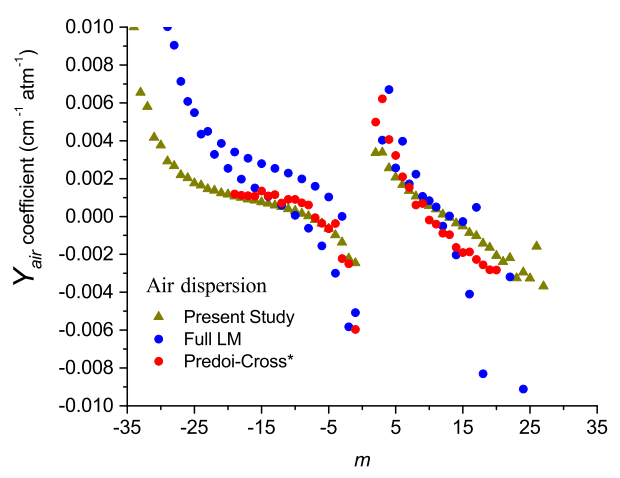

) 
The temperature dependences of the pressure shift parameters $\left(\delta_{X}^{\prime}\right)$ are found to be significantly determinable and indicate that the pressure shifts become more negative at decreasing temperatures in the atmospheric temperature range. The values are depicted in $12 \mathrm{c}$ and $12 \mathrm{~d}$ and have units of $\mathrm{cm}^{-1} / \mathrm{atm} / \mathrm{K}(0.29587215 \mathrm{MHz} / \mathrm{Pa} / \mathrm{K})$ with using the linear temperature dependence expression of Eq. 6. The temperature dependence of the nitrogen shift was found to start near zero at low $J$ and increase monotonically throughout the determinable range, above $J=23$ the parameters were fixed to a constant value of $5 \times 10^{-5} \mathrm{~cm}^{-1} \mathrm{~atm}^{-1} \mathrm{~K}^{-1}$. For oxygen pressure shift temperature dependence the values initially rise at small $J$ and then level off around $6 \times 10^{-5} \mathrm{~cm}^{-1} \mathrm{~atm}^{-1} \mathrm{~K}^{-1}$, values up $J=23$ were fixed to $3 \times 10^{-5}$ $\mathrm{cm}^{-1} \mathrm{~atm}^{-1} \mathrm{~K}^{-1}$.

The unitless speed dependence parameters $(S)$ are shown in Figure 12e. As expected the values are near 0.1 across the band as is typical for oxygen and nitrogen mixtures. Since speed-dependence is expected to be constant across a band and a trend towards slightly larger $S$ values is observed on increasing $J$, attempts were made to also determine the heavily correlated motional (Dicke) narrowing. Long et al. [14] fitted Dicke narrowing, rather than speed-dependence, and trial fits were performed using either parameterization, as well as both simultaneously. Fitting both parameters produced divergent solutions. Fitting only one parameter at a time revealed that the CRDS data itself was unaffected by the choice, but that the FTS data had poorer residuals when fitted with Dicke narrowing alone. Certainly the intermediate pressure range of the FTS data, as well as unsaturated line centers, presents a stronger test for the effect. Finally, several tests were performed with speed-dependence fixed and Dicke narrowing optimized, these tests revealed poorer fits than with speed-dependence alone, and may be an indication that the algorithm in use for this case is not sufficiently accurate. Work is currently underway to integrate the Hartmann-Tran profile [52] into the program and these tests will then be repeated.

Line-mixing is often condensed to the line-by-line dependent dispersion coefficient, $Y$ at a given temperature for tabulation and use in LBL codes (see Eqn. 9). Previously, $Y$ factors from $-20 \leq m \leq 20$ were fitted at room temperature for both self-perturbed $\mathrm{O}_{2}$ [12] and nitrogen perturbed $\mathrm{O}_{2}$ [13] and these were used to guide Tran and Hartmann's empirical correction to their $\mathbf{W}$-matrix [36]. In that study, all of the off-diagonal elements (odd and even $\left.\Delta J_{\mathrm{s}}\right)$ were scaled between 0 and 1 with line-mixing for small quanta obtaining larger relative values and line-mixing for large quanta scaling to 
near zero, and $J$ values around 10 , near the room temperature $P / R$ maxima, were scaled by $\sim \frac{1}{2}$. In Figure $12 \mathrm{f}$ the values from the present study (with no $\Delta J$ odd elements and a constant empirical scaling of $\frac{1}{2}$ ) are compared to unscaled values from the full $\mathbf{W}$-matrix provided by Ref. [22] as well as air values calculated from the experimentally determined room-temperature values reported by Predoi-Cross et al. [12, 13]. Several features evident in Figure $12 \mathrm{f}$ are worth noting (1) the bifurcation of the $Y$ points between even and odd $J$, that was not observed in fitted $Y$-values of Refs. [12, 13] disappears entirely upon removal of the odd $\Delta J$ matrix elements; (2) the $J$ dependences of the curves are all of correct sign and cross through each other at the strongest portions of the sub-bands; (3) the empirical factor of $\frac{1}{2}$ applied in the present study tracks well with fitted values $[12,13]$ where they are available. The need for $J$-dependent empirical scaling in Ref. [36] was deemed to be a consequence of the assumptions about case $a$ intensities in the matrix element scale factors, however these errors are largely of just a few $\%$, and the empirical corrections amount to 0-100\% scaling, which also significantly reduced couplings in the band-head region where many lines are accidentally degenerate. The removal of odd $\Delta J$ elements in this work accomplishes significant reductions of couplings in the band-head region and allows a scalar to accommodate matching of the LM model. Implementation of the full $\mathbf{W}$-matrix, without $J$-dependent empirical scaling, retains a strong link to the fundamental collisional theory and provides for a well-defined temperature dependent model of the LM. These modified matrices, as well as $Y$ values determined for air at a variety of temperatures, are provided in the supplemental material.

\subsection{Testing in OCO-2 Level 2 Algorithm}

A multi-dimensional table containing the various cross-sections of $\mathrm{O}_{2}$ Aband absorption with the dependent variables of temperature, air pressure and water pressure is produced using the results of the fitting to laboratory and atmospheric data. The table incorporates both resonant and nonresonant absorption as well as the interfering species $\mathrm{H}_{2} \mathrm{O}$ which is also allowed to be a foreign broadener [26]. It is generated using the physical model portion of the multispectral fitting program as part of the compiled code. The resulting table is one of the absorption coefficient (ABSCO) tables that are primary inputs to the ACOS (Level 2) Algorithm [5]. At the time of preparing and testing this table, the OCO-2 mission has acquired more than one-year of data on orbit and subsets of the data analyzed using the 
seventh version of the Level 2 code (v7.1, which uses ABSCO table v4.2) were identified for diverse atmospheric conditions, minimal aerosol loading, and cloud-free observations.

To compare and test the new ABSCO table several physically relevant aspects of the level-2 products are tracked including the averaged spectral residuals, surface pressure and impact on aerosol retrieval. Since a set of empirically generated equivalent orthogonal functions (EOFs) are introduced to remove persistent spectral residuals in v7.1, a special processing of this version of the algorithm, in which no EOFs are applied, was run to enable the spectral residuals to be 'true' and directly comparable to tests with the new spectral data.

The results of the testing are somewhat mixed: 1) there is a clear improvement in the dependence of the derived surface pressure as compared to ground truth measurements; 2) the airmass dependence of the derived surface pressure is strongly reduced; 3) persistent and slowly varying spectral residual features extending across the $P$ and $R$ branches disappeared, but new sharp, localized features appeared between the spin-doublets; 4) aerosol loading (at the small values selected for these tests) is not noticeably affected. These results are encouraging, indicating that the airmass dependence of the derived surface pressure had been linked to an imbalance between the effects of LM and CIA, which are carefully balanced as well as possible in the new ABSCO table. The spectral residual changes reflect the band-wide change in intensity that has resulted from this new balance, which has effectively made the central regions of the $P, R$ branches stronger at high pressure, and reduced the amount of absorption in the high $J, P$-branch. The residuals near the band-head are different, and the abrupt transition from resonant to non-resonant absorption in this area is actually slightly worse than the prior ABSCO table (v4.2). The most disconcerting result of the test is the appearance of regular residuals between the spin doublets. These features are also discernable in the CRDS $P$-branch data, and further modeling of the $\Delta J$ odd LM interactions has been shown to reduce these features, but there is not currently enough information in the data sets described here to reliably balance this additional (and weaker) LM with the CIA. Overall the change in residuals is a net reduction (in the root-mean-square), and an ad hoc scaling parameter that was previously necessary to minimize surface pressure bias in ABSCO v4.2 is now unnecessary. The remaining spectral residuals will likely be treated with new EOFs in future level 2 processing. 


\section{Conclusion}

This work represents the first concerted effort to treat $\mathrm{O}_{2}$ LM,CIA and non-Voigt lineshapes on temperature dependent laboratory data. The multispectral fitting program was modified as necessary to accommodate the combination of FTS and CRDS data, as well as to incorporate approximate CIA while determining LBL and adjusting LM. Complementary data from both FTS and CRDS in the $P$-branch improved atmospheric models directly, while atmospheric datasets were essential to proper tuning of the LM and CIA in the $R$-branch. Besides a scale factor of $1 / 2$ on the LM, the LM in the $R$-branch closely resembles that of Tran et al. [22] with the absence of coupling between sub-bands. LBL parameters closely resemble those of HITRAN2012 and its data sources. Together the derived LBL, LM and CIA provide a self-consistent parameter set, traceable to calibrated lab and atmospheric data, for the production of new ABSCO tables. The use of an improved ABSCO table in the retrieval of atmospheric trace gas concentrations reduces band-wide residuals in the spectral data and reduces biases of the surface pressure associated with systematic errors in previous spectroscopic data sources. The ABSCO table from this work (version 5.0) is available upon request along with those associated with $\mathrm{CO}_{2}$ absorption. An update to LBLRTM [63] that incorporates the results of this work will also be available.

This effort has reduced biases and improved retrievals of atmospheric data, however, it is clear that some LM/CIA issues remain in the high density models. Efforts are currently underway to record reduced temperature CRDS data in this spectral region, including the $R$-branch, and a photo-acoustic spectrometer at California Institute of Technology [64] has been refitted for low-temperature measurements as well. The expected datasets will provide much needed data at high density for both long (CRDS) and short (PAS) pathlengths.

\section{Acknowledgments}

Portions of the research described in this paper were performed at the Jet Propulsion Laboratory, California Institute of Technology, under contract with the National Aeronautics and Space Administration. Copyright 2016 California Institute of Technology. Government sponsorship acknowledged. Research performed at the College of William and Mary was supported by a 
Grant from the Jet Propulsion Laboratory, California Institute of Technology. The authors gratefully acknowledge financial support from the U.S. Department of Energy, Office of Science, Office of Biological and Environmental Research, Environmental Sciences Division, as part of the ARM programme under grant DE-FG02-90ER610. Support was also provided by the National Institute of Standards and Technology (NIST) Greenhouse Gas Measurements and Climate Research Program and the Orbiting Carbon Observatory (OCO) project, a National Aeronautics and Space Administration (NASA) Earth System Science Pathfinder (ESSP) mission. Manufacturers and product names are listed solely for completeness. These specific citations neither constitute an endorsement of the products nor imply that similar products from other companies would be less suitable. We thank Dr. Ha Tran for providing line mixing coefficients for pure $\mathrm{O}_{2}$. We also thank Dr. Debra Wunch for providing TCCON spectra and guidance on good days for use on our analysis.

\section{References}

\section{References}

[1] L. S. Rothman, A. Barbe, D. C. Benner, L. R. Brown, C. Camy-Peyret, M. R. Carleer, et al., The HITRAN Molecular Spectroscopic Database: Edition Of 2000 Including Updates Through 2001, J. Quant. Spectrosc. Radiat. Transfer 82 (1) (2003) 5-44.

[2] L. S. Rothman, D. Jacquemart, A. Barbe, D. C. Benner, M. Birk, L. R. Brown, et al., The HITRAN 2004 Molecular Spectroscopic Database, J. Quant. Spectrosc. Radiat. Transfer 96 (2) (2005) 139-204.

[3] L. S. Rothman, I. E. Gordon, A. Barbe, D. C. Benner, P. E. Bernath, M. Birk, et al., The HITRAN 2008 Molecular Spectroscopic Database, J. Quant. Spectrosc. Radiat. Transfer 110 (9-10) (2009) 533-572.

[4] A. Eldering, S. Boland, B. Solish, D. Crisp, P. Kahn, M. Gunson, High Precision Atmospheric $\mathrm{CO}_{2}$ Measurements From Space: The Design And Implementation Of OCO-2, Aerospace Conference, 2012 IEEE (2012) 1-10.

[5] C. O’Dell, B. Connor, H. Bösch, D. O'Brien, C. Frankenberg, R. Castano, et al., The ACOS $\mathrm{CO}_{2}$ Retrieval Algorithm Part 1: Description 
and Validation Against Synthetic Observations, Atmos. Meas. Tech. Discuss. 4 (5) (2011) 6097-6158. doi:10.5194/amtd-4-6097-2011.

[6] C. W. O'Dell, B. Connor, H. Bösch, D. O'Brien, C. Frankenberg, R. Castano, et al., The ACOS $\mathrm{CO}_{2}$ Retrieval Algorithm - Part 1: Description And Validation Against Synthetic Observations, Atmos. Meas. Tech. 5 (1) (2012) 99-121. doi:10.5194/amt-5-99-2012.

[7] D. Crisp, B. M. Fisher, C. O’Dell, C. Frankenberg, R. Basilio, H. Bösch, et al., The ACOS $\mathrm{CO}_{2}$ Retrieval Algorithm - Part II: Global X$\mathrm{CO}_{2}$ Data Characterization, Atmos. Meas. Tech. 5 (4) (2012) 687-707. doi:10.5194/amt-5-687-2012.

[8] D. Crisp, H. Bösch, L. R. Brown, R. Castano, M. Christi, B. Connor, et al., OCO (Orbiting Carbon Observatory)-2 Level 2 Full Physics Retrieval Algorithm Theoretical Basis, Tech. Rep. OCO D-65488, NASA Jet Propulsion Laboratory, California Institute of Technology, Pasadena, CA, version $1.0 \mathrm{Rev}$ 4, http : //disc.sci.gsfc.nasa.gov/acdisc/documentation/OCO $2_{L} 2_{F} P_{A} T B D_{v} 1_{r} e v 4_{N}$ ov10.pdf , (last access: January 2012).

[9] D. R. Thompson, D. Chris Benner, L. R. Brown, D. Crisp, V. Malathy Devi, Y. Jiang, et al., Atmospheric Validation of High Accuracy $\mathrm{CO}_{2}$ Absorption Coefficients for the OCO-2 Mission, J. Quant. Spectrosc. Radiat. Transfer 113 (17) (2012) 2265-2276. doi:http://dx.doi.org/10.1016/j.jqsrt.2012.05.021.

[10] A. Kuze, H. Suto, M. Nakajima, T. Hamazaki, Thermal And Near Infrared Sensor For Carbon Observation Fourier-Transform Spectrometer On The Greenhouse Gases Observing Satellite For Greenhouse Gases Monitoring, Appl. Opt. 48 (35) (2009) 6716-6733.

[11] D. Wunch, G. C. Toon, P. O. Wennberg, S. C. Wofsy, B. B. Stephens, M. L. Fischer, et al., Calibration Of The Total Carbon Column Observing Network Using Aircraft Profile Data, Atmos. Meas. Tech. 3 (5) (2010) 1351-1362.

[12] A. Predoi-Cross, K. Hambrook, R. Keller, C. Povey, I. Schofield, D. Hurtmans, H. Over, G. C. Mellau, Spectroscopic Lineshape Study 
of the Self-Perturbed Oxygen A-Band, J. Mol. Spectrosc. 248 (2008) $85-110$.

[13] A. Predoi-Cross, C. Holladay, H. Heung, J.-P. Bouanich, G. C. Mellau, R. Keller, D. R. Hurtmans, Nitrogen-Broadened Lineshapes in the Oxygen A-Band: Experimental Results and Theoretical Calculations, J. Mol. Spectrosc. 251 (2008) 159-175.

[14] D. A. Long, D. K. Havey, M. Okumura, C. E. Miller, J. T. Hodges, $\mathrm{O}_{2}$ A-Band Line Parameters To Support Atmospheric Remote Sensing, J. Quant. Spectrosc. Radiat. Transfer 111 (14) (2010) 2021-2036. doi:10.1016/j.jqsrt.2010.05.011.

[15] D. J. Robichaud, J. T. Hodges, L. R. Brown, D. Lisak, P. Masłowski, L. Y. Yeung, et al., Experimental Intensity and Lineshape Parameters of the Oxygen A-Band using Frequency-Stabilized Cavity Ring-Down Spectroscopy, J. Mol. Spectrosc. 248 (1) (2008) 1 - 13. doi:10.1016/j.jms.2007.10.010.

[16] D. A. Long, D. K. Havey, S. S. Yu, M. Okumura, C. E. Miller, J. T. Hodges, $\mathrm{O}_{2}$ A-Band Line Parameters To Support Atmospheric Remote Sensing. Part II: The Rare Isotopologues, J. Quant. Spectrosc. Radiat. Transfer 112 (16) (2011) 2527-2541. doi:10.1016/j.jqsrt.2011.07.002.

[17] D. J. Robichaud, J. T. Hodges, D. Lisak, C. E. Miller, M. Okumura, High-Precision Pressure Shifting Measurement Technique Using Frequency-Stabilized Cavity Ring-Down Spectroscopy, J. Quant. Spectrosc. Radiat. Transfer 109 (3) (2008) 435-444.

[18] D. A. Long, D. K. Havey, M. Okumura, H. M. Pickett, C. E. Miller, J. T. Hodges, Laboratory Measurements And Theoretical Calculations Of $\mathrm{O}_{2}$ A-Band Electric Quadrupole Transitions, Phys. Rev. A 80 (4) (2009) 12. doi:10.1103/PhysRevA.80.042513.

[19] D. K. Havey, D. A. Long, M. Okumura, C. E. Miller, J. T. Hodges, Ultra-Sensitive Optical Measurements Of High-J Transitions in the $\mathrm{O}_{2}$ A-Band, Chem. Phys. Lett. 483 (1-3) (2009) 49-54. doi:10.1016/j.cplett.2009.10.067.

[20] D. Lisak, P. Maslowski, A. Cygan, K. Bielska, S. Wojtewicz, M. Piwinski, et al., Line Shapes and Intensities of Self-Broadened 
$\mathrm{O}_{2} b^{1} \Sigma_{g}^{+}(\nu=1) \leftarrow X^{3} \Sigma_{g}^{-}(\nu=0)$ Band Transitions Measured By Cavity Ring-Down Spectroscopy, Phys. Rev. A 81 (4) (2010) 10. doi:10.1103/PhysRevA.81.042504.

[21] L. R. Brown, C. Plymate, Experimental Line Parameters Of The Oxygen A-Band At 760 nm, J. Mol. Spectrosc. 199 (2) (2000) 166-179.

[22] H. Tran, C. Boulet, J. M. Hartmann, Line Mixing And Collision-Induced Absorption By Oxygen In The A-Band: Laboratory Measurements, Model, And Tools For Atmospheric Spectra Computations, J. Geophys. Res. D: Atmos 111 (D15). doi:10.1029/2005jd006869.

[23] D. A. Long, D. J. Robichaud, J. T. Hodges, Frequency-Stabilized Cavity Ring-Down Spectroscopy Measurements Of Line Mixing And CollisionInduced Absorption In The $\mathrm{O}_{2}$ A-Band, J. Chem. Phys. 137 (1) (2012) 014307. doi:10.1063/1.4731290.

[24] E. Vess, C. Wallace, H. Campbell, V. Awadalla, J. Hodges, D. Long, et al., Measurement Of $\mathrm{H}_{2} \mathrm{O}$ Broadening Of $\mathrm{O}_{2}$ A-Band Transitions And Implications For Atmospheric Remote Sensing, J. Phys. Chem. A 116 (16) (2012) 4069-4073.

[25] G. Fanjoux, G. Millot, R. Saint-Loup, R. Chaux, R. L., Coherent antiStokes Raman spectroscopy study of collisional broadening in the $\mathrm{O}_{2}$ $\mathrm{H}_{2} \mathrm{O}$ Q branch, J. Chem. Phys. 101 (1994) 1061-1071.

[26] B. J. Drouin, V. Payne, F. Oyafuso, K. Sung, E. Mlawer, Pressure Broadening of Oxygen by Water, J. Quant. Spectrosc. Radiat. Transfer 133 (2014) 190-198.

[27] G. Y. Golubiatnikov, M. A. Koshelev, A. F. Krupnov, Reinvestigation Of Pressure Broadening Parameters At 60-GHz Band And Single 118.75 GHz Oxygen Lines At Room Temperature, J. Mol. Spectrosc. 222 (2) (2003) 191-197. doi:10.1016/j.jms.2003.08.002.

[28] L. S. Rothman, I. E. Gordon, Y. Babikov, A. Barbe, D. Chris Benner, P. F. Bernath, et al., The HITRAN 2012 Molecular Spectroscopic Database, J. Quant. Spectrosc. Radiat. Transfer 130 (0) (2013) 4-50. doi:10.1016/j.jqsrt.2013.07.002. 
[29] F. R. Spiering, M. B. Kiseleva, N. N. Filippov, H. Naus, B. van Lieshout, C. Weijenborg, et al., Line Mixing And Collision Induced Absorption In The Oxygen A-Band Using Cavity Ring-Down Spectroscopy, The J. Chem. Phys. 133 (11) (2010) 114305.

[30] D. A. Long, J. T. Hodges, On Spectroscopic Models Of The $\mathrm{O}_{2}$ A-Band And Their Impact Upon Atmospheric Retrievals, J. Geophys. Res. D: Atmos. 117 (D12). doi:10.1029/2012jd017807.

[31] N. Moazzen-Ahmadi, J. N. Oliaee, I. Ozier, E. Wishnow, K. Sung, T. Crawford, et al., An Intensity Study Of The Torsional Bands Of

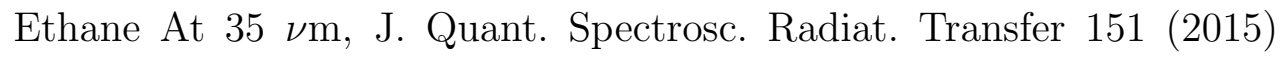
123-132.

[32] E. Wishnow, A. Leung, H. Gush, Cryogenic Multiple Reflection Absorption Cell And Fourier Transform Spectrometer System For The Far Infrared, Rev. Sci. Instrum. 70 (1) (1999) 23-31.

[33] D. D. Turner, S. A. Clough, J. C. Lijegren, E. E. Clothiaux, K. E. CadyPereira, K. L. Gaustad, Retrieving Liquid Water Path And Precipitable Water Vapor From The Atmospheric Radiation Measurement (ARM) Microwave Radiometers, IEEE Trans. Geosci. Remote Sens. 45 (11) (2007) 3680-3690. doi:10.1109/tgrs.2007.903703.

[34] D. C. Benner, C. P. Rinsland, V. M. Devi, M. A. H. Smith, D. Atkins, A Multispectrum Nonlinear Least-Squares Fitting Technique, J. Quant. Spectrosc. Radiat. Transfer 53 (1995) 705-21.

[35] S. Falke, E. Tiemann, C. Lisdat, H. Schnatz, G. Grosche, Transition Frequencies Of The D Lines Of ${ }^{39} \mathrm{~K},{ }^{40} \mathrm{~K}$, And ${ }^{41} \mathrm{~K}$ Measured With A Femtosecond Laser Frequency Comb, Phys. Rev. A 74 (3) (2006) 9. doi:10.1103/PhysRevA.74.032503.

[36] H. Tran, J. M. Hartmann, An improved O-2 A-Band Absorption Model and its Consequences for Retrievals of Photon Paths and Surface Pressures, J. Geophys. Res. D: Atmos 113 (D18). doi:10.1029/2008JD010011.

[37] A. E. DePristo, S. D. Augustin, R. Ramaswamy, H. Rabitz, Quantum Number and Energy Scaling for Nonreactive Collisions, The Journal of Chemical Physics 71 (2) (1979) 850-865. 
[38] F. Niro, C. Boulet, J. Hartmann, Spectra calculations in central and wing regions of $\mathrm{CO}_{2}$ IR bands between 10 and $20 \mu \mathrm{m}$. I: model and laboratory measurements, J. Quant. Spectrosc. \& Radiat. Trans. 88 (4) (2004) 483-498. doi:10.1016/j.jqsrt.2004.04.003.

[39] P. W. Rosenkranz, Shape of $5 \mathrm{~mm}$ Oxygen Band in Atmosphere, IEEE Trans. Antennas Propag. AP23 (4) (1975) 498-506.

[40] S. S. Yu, B. J. Drouin, C. E. Miller, High Resolution Spectral Analysis Of Oxygen IV. Energy Levels, Partition Sums, Band Constants, RKR Potentials, Franck-Condon Factors Involving The $\mathrm{X}{ }^{3} \Sigma_{g}^{-}$, a ${ }^{1} \Delta_{g}$, and b ${ }^{1} \Sigma_{g}^{+}$States, J. Chem. Phys. 141 (17) (2014) 12. doi:10.1063/1.4900510.

[41] S. S. Yu, C. E. Miller, B. J. Drouin, H. S. P. Muller, High Resolution Spectral Analysis Of Oxygen I. Isotopically Invariant Dunham Fit For The $\mathrm{X}^{3} \Sigma_{g}^{-}$, a ${ }^{1} \Delta_{g}$, b ${ }^{1} \Sigma_{g}^{+}$States, J. Chem. Phys. 137 (2) (2012) 20. doi:10.1063/1.4719170.

[42] B. J. Drouin, H. Gupta, S. Yu, C. E. Miller, H. S. P. Muller, High Resolution Spectral Analysis of Oxygen. II. Rotational Spectra of $a^{1} \Delta_{g} O_{2}$ Isotopologues, J. Chem. Phys. 137 (2) (2012) 11. doi:10.1063/1.4719169.

[43] B. J. Drouin, S. Yu, B. M. Elliott, T. J. Crawford, C. E. Miller, High Resolution Spectral Analysis of Oxygen. III Laboratory Investigation of the Airglow Bands, J. Chem. Phys. 139 (14) (2013) 11. doi:10.1063/1.4821759.

[44] J. Watson, Rotational Line Intensities in ${ }^{3} \Sigma^{-}{ }^{1} \Sigma$ Electronic Transitions, Can. J. Phys. 46 (14) (1968) 1637-\&.

[45] R. Herman, R. F. Wallis, Influence of Vibration-Rotation Interaction on Line Intensities in Vibration-Rotation Bands of Diatomic Molecules, The J. Chem. Phys. 23 (4) (1955) 637-646.

[46] A. S. Pine, J. P. Looney, $\mathrm{N}_{2}$ and Air Broadening in the Fundamental Bands of HF and HCl., J. Mol. Spectrosc. 122 (1987) 41-55.

[47] R. Ciurylo, Shapes of Pressure- and Doppler- Broadened Spectral Lines in the Core and Near Wings, Phys. Rev. A 58 (1998) 1029-1039. 
[48] F. Rohart, H. Mäder, H. W. Nicolaisen, Speed Dependence of Rotational Relaxation Induced by Foreign Gas Collisions: Studies of $\mathrm{CH}_{3} \mathrm{~F}$ by Millimeter Wave Coherent Transients, J. Chem. Phys. 101 (1994) 6475-6488.

[49] D. Priem, F. Rohart, J. M. Colmont, G. Wlodarczak, J. P. Bouanich, Lineshape study of the $J=3 \leftarrow 2$ rotational transition of CO perturbed by $\mathrm{N}_{2}$ and $\mathrm{O}_{2}$, J. Mol. Struct. 517-518 (2000) 435-454.

[50] K. L. Letchworth, D. C. Benner, Rapid And Accurate Calculation Of The Voigt Function, J. Quant. Spectrosc. Radiat. Transfer 107 (1) (2007) 173-192.

[51] L. S. Rothman, I. E. Gordon, et al., HITRANonline, J. Quant. Spectrosc. Radiat. Transfer.

[52] H. Tran, N. H. Ngo, J. M. Hartmann, Efficient Computation of Some Speed-Dependent Isolated Line Profiles, J. Quant. Spectrosc. Radiat. Transfer 129 (2013) 199-203. doi:10.1016/j.jqsrt.2013.06.015.

[53] J. M. Hartmann, C. Boulet, D. Robert, Collisional Effects On Molecular Spectra, 1st Edition, Elsevier, Amsterdam, 2008.

[54] A. Lévy, N. Lacome, C. Chackerian, Collisional Line Mixing in Spectroscopy of the Earths Atmosphere and Interstellar medium, K. Narahari Rao and A. Weber, (Academic Press, Boston, MA, 1992), Academic Press, Boston, MA, 1992.

[55] G. N. Lewis, The Magnetism of Oxygen and the Molecule $\mathrm{O}_{4}$, J. Am. Chem. Soc. 46 (9) (1924) 2027-2032.

[56] B. Maté, C. Lugez, G. T. Fraser, W. J. Lafferty, Absolute Intensities for the $\mathrm{O}_{2} 1.27 \mu \mathrm{m}$ Continuum Absorption, J. Geophys. Res. 104 (1999) 30,585 .

[57] M. J. Alvarado, V. H. Payne, E. J. Mlawer, G. Uymin, M. W. Shephard, K. E. Cady-Pereira, et al., Performance of the Line-By-Line Radiative Transfer Model (LBLRTM) for Temperature, Water Vapor, and Trace Gas Retrievals: Recent Updates Evaluated with IASI Case Studies, Atmos. Chem. Phys. 13 (14) (2013) 6687-6711. doi:10.5194/acp-13-66872013. 
[58] F. R. Spiering, M. B. Kiseleva, N. N. Filippov, B. van Lieshout, A. M. H. van der Veen, W. J. van der Zande, The Effect Of Collisions With Nitrogen On Absorption By Oxygen In The A-Band Using Cavity Ring-Down Spectroscopy, Mol. Phys. 109 (4) (2011) 535-542. doi:10.1080/00268976.2010.533709.

[59] F. Lique, Temperature Dependence of the Fine-Structure Resolved Rate Coefficients for collisions of $\mathrm{O}_{2}\left(X^{3} \Sigma_{g}^{-}\right)$with He, J. Chem. Phys. 132 (4). doi:10.1063/1.3299283.

[60] D. A. Long, D. K. Havey, M. Okumura, H. M. Pickett, C. E. Miller, J. T. Hodges, Laboratory Measurements and Theoretical Calculations of $\mathrm{O}_{2}$ A-Band Electric Quadrupole Transitions, Phys. Rev. A 80 (2009) 042513.

[61] R. Schermaul, R. C. M. Learner, Precise Line Parameters and Transition Probability of the Atmospheric A Band of Molecular Oxygen ${ }^{16} \mathrm{O}_{2}$, J. Quant. Spectrosc. Radiat. Transfer 61 (1999) 781-794.

[62] B. Connor, Personal Communication, 2015.

[63] S. A. Clough, M. W. Shephard, E. J. Mlawer, J. S. Delamere, M. J. Iacono, K. Cady-Pereira, S. Boukabara, P. D. Brown, Atmospheric radiative transfer modeling: a summary of the AER codes, J. Quant. Spectrosc. Radiat. Transfer 91 (2005) 233-244.

[64] T. Bui, Cavity-enhanced spectroscopies for applications of remote sensing, chemical kinetics and detection of radical species, Ph. D. Thesis, California Institute of Technology.

[65] H. M. Pickett, The Fitting And Prediction Of Vibration-Rotation Spectra With Spin Interactions, J. Mol. Spectrosc. 148 (2) (1991) 371-377.

[66] U. I. Safronova, M. S. Safronova, High-Accuracy Calculation Of Energies, Lifetimes, Hyperfine Constants, Multipole Polarizabilities, And Blackbody Radiation Shift in ${ }^{39}$ K, Phys. Rev. A 78 (5) (2008) 052504.

[67] J. R. de Laeter, J. K. Böhlke, P. De Biévre, H. Hidaka, H. Peiser, K. Rosman, et al., Atomic Weights Of The Elements. Review 2000 (IUPAC Technical Report), Pure Appl. Chem. 75 (6) (2003) 683-800. 
[68] G. Audi, A. H. Wapstra, T. C, The 2003 Mass Evaluation: (II). Tables, Graphs and References, Nuc. Phys. A. 729(1) (2003) 337-676.

\section{Supplemental Material}



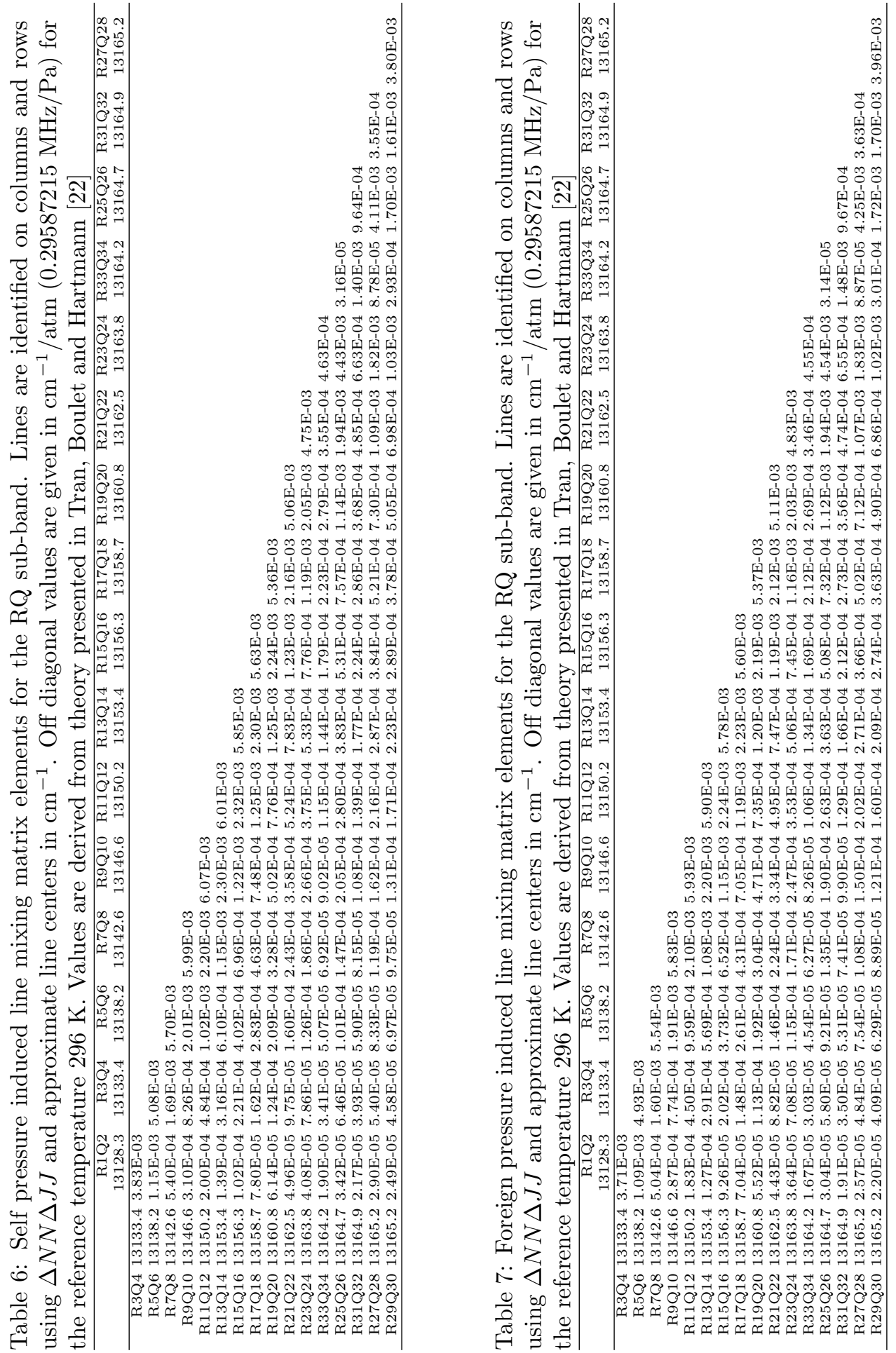

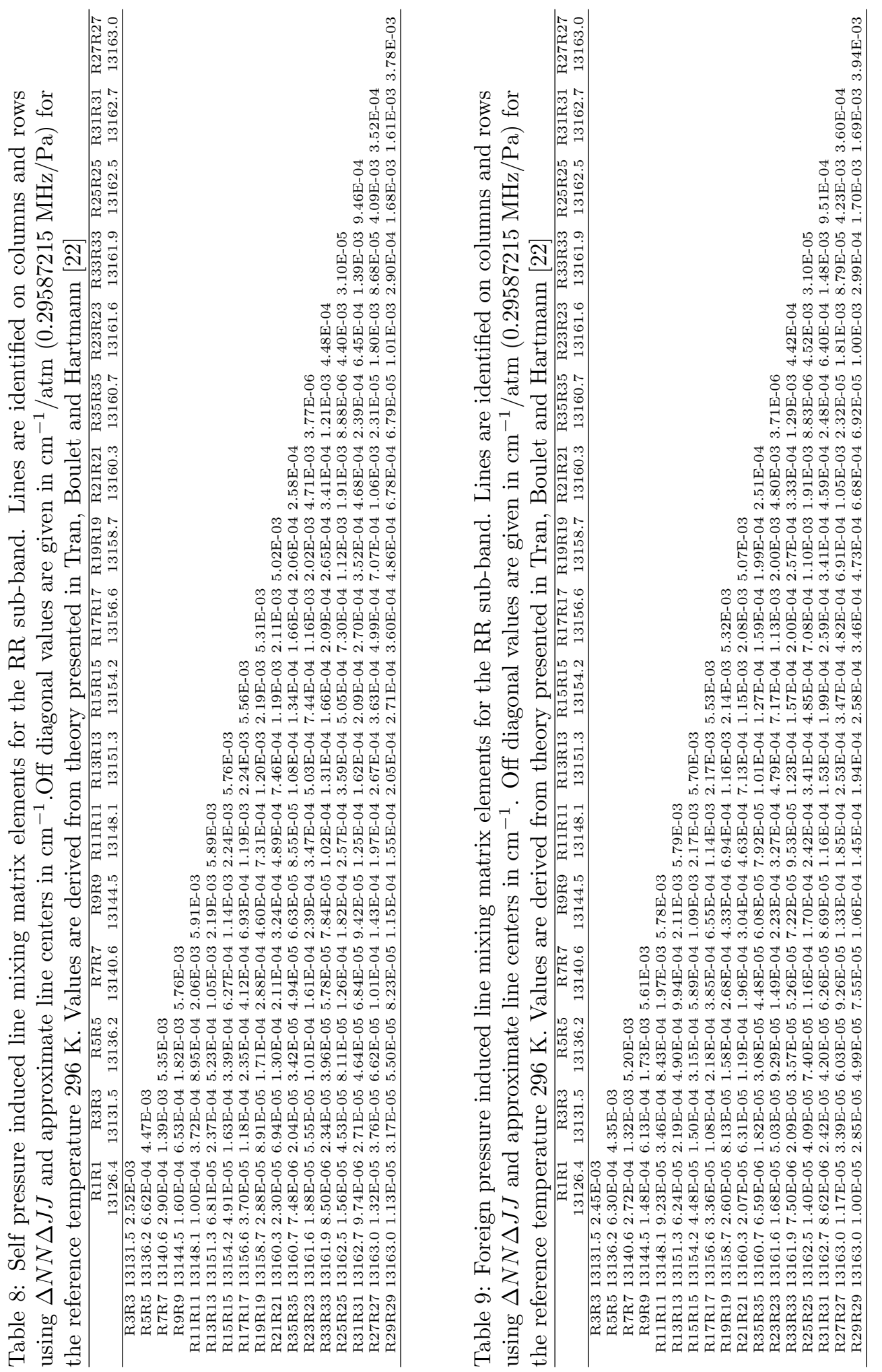

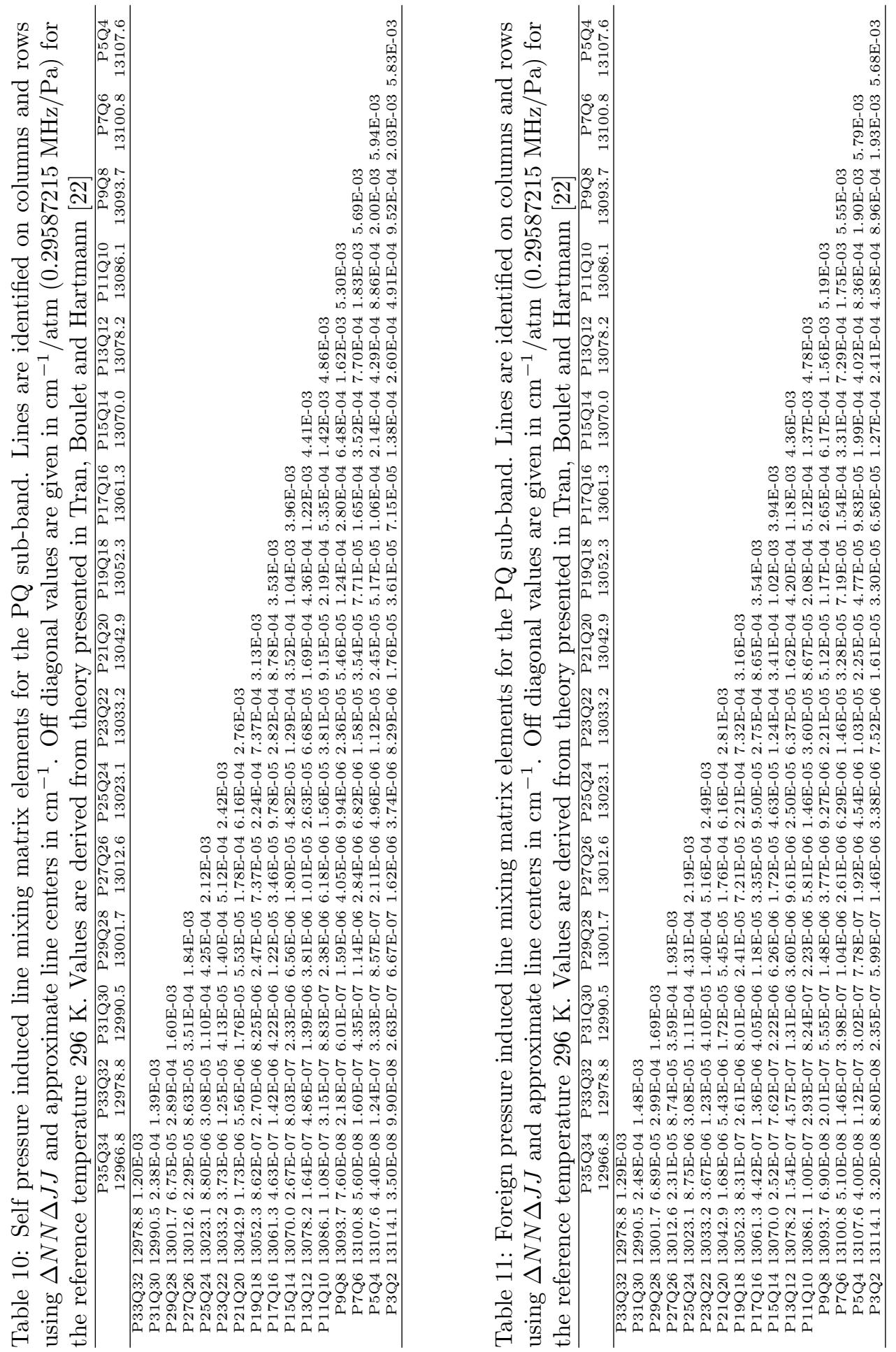

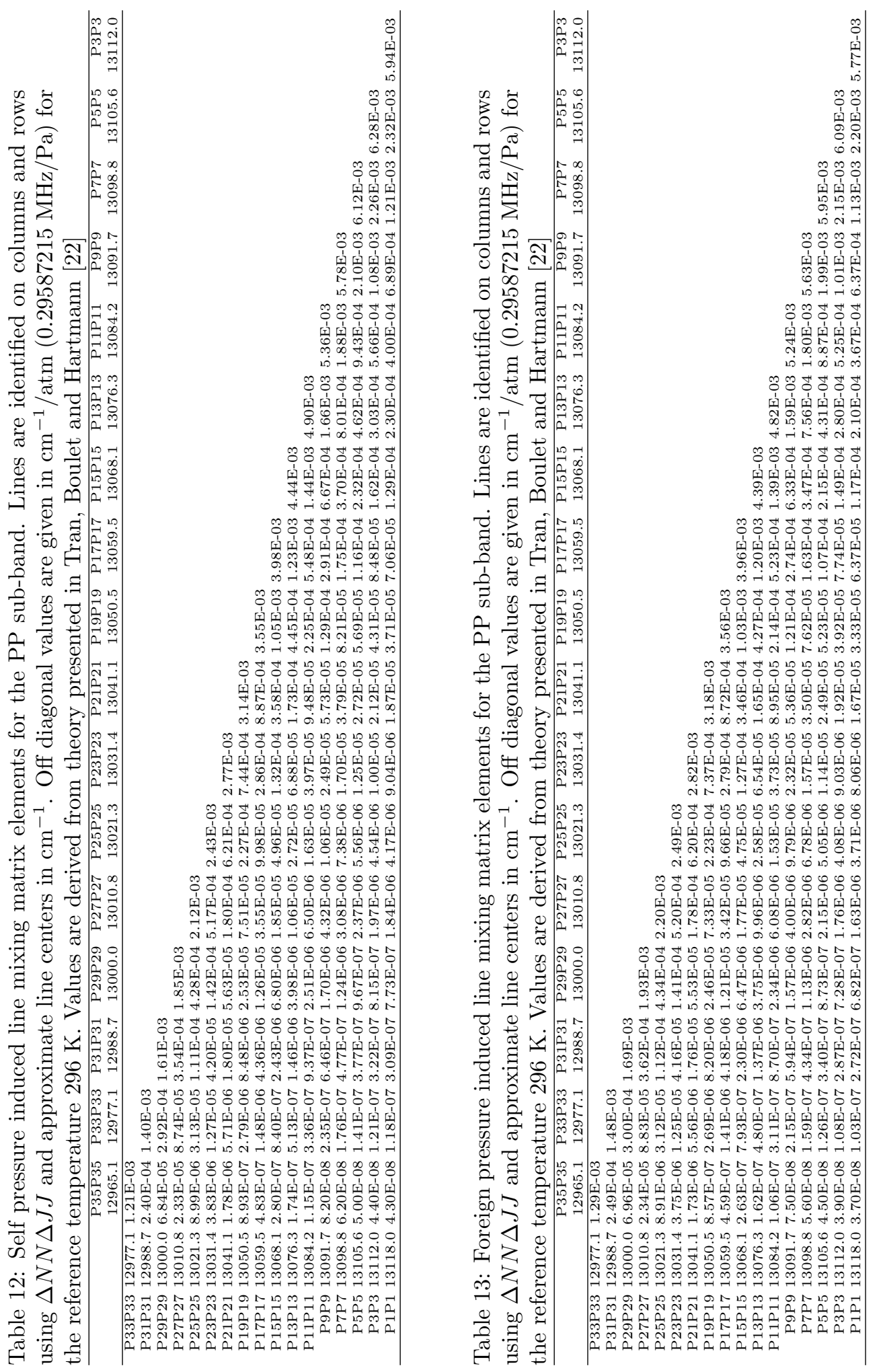

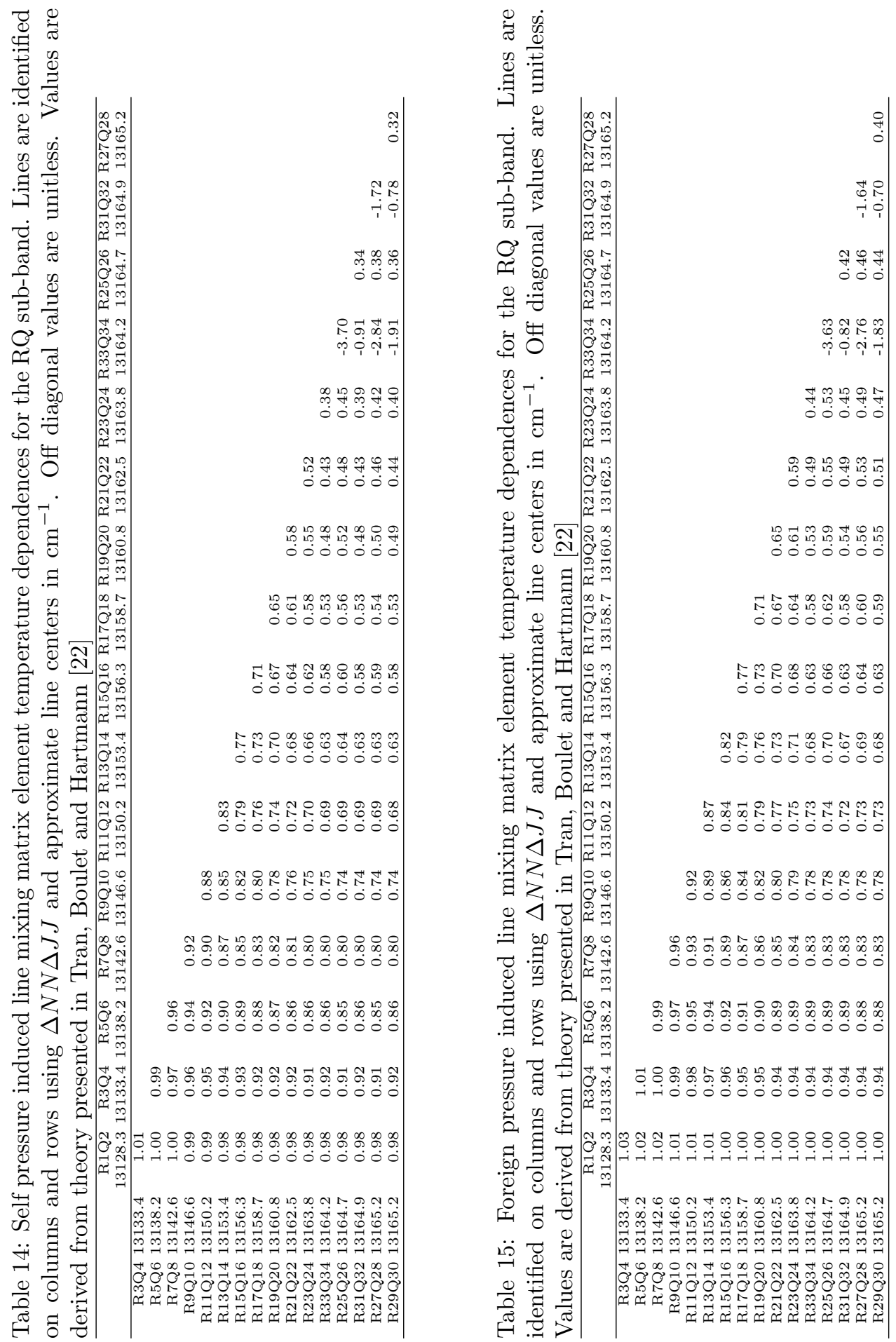

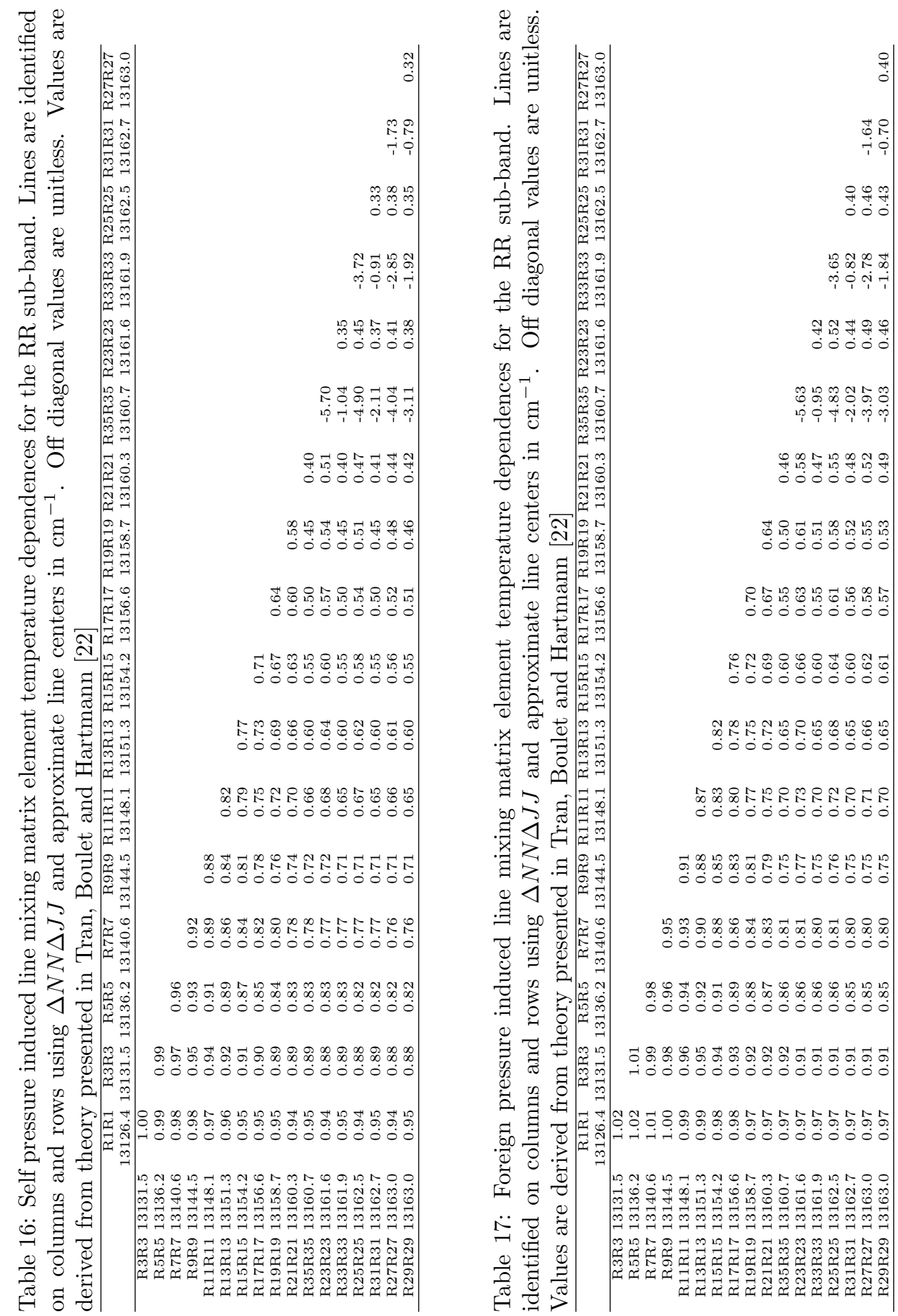

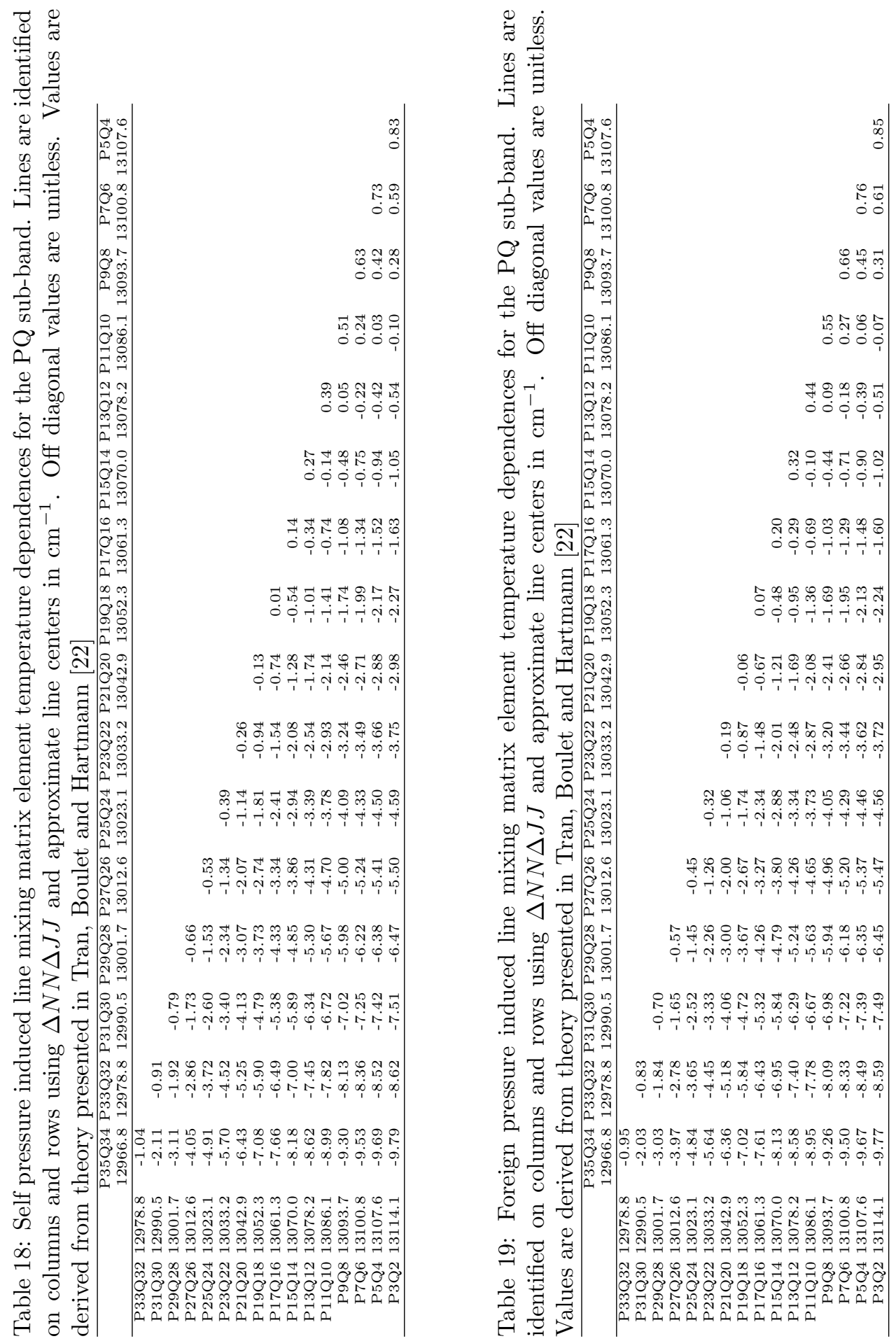

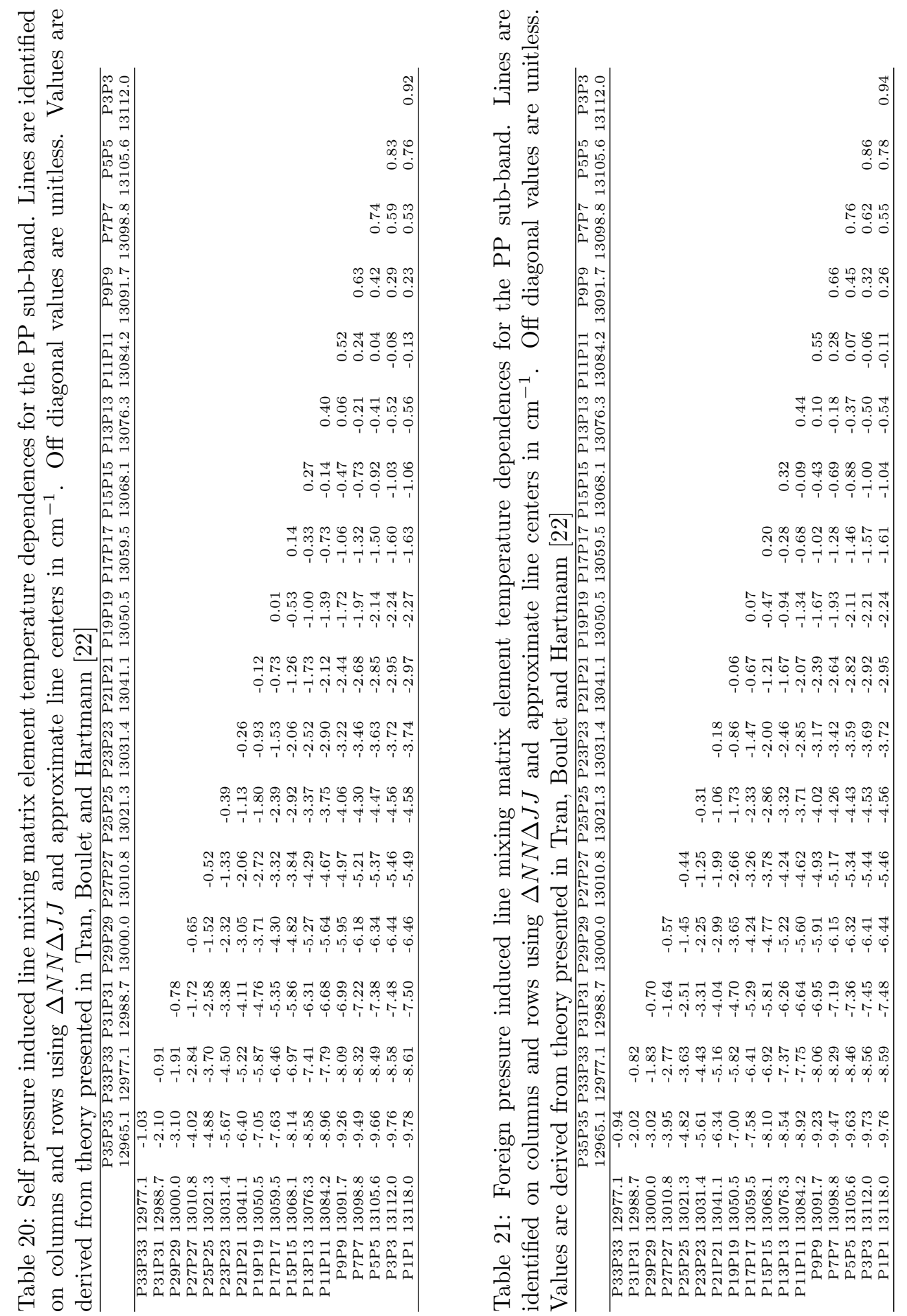
Air line mixing matrix elements may be calculated for specific temperatures and pressures and the post-diagonalization Y-factors tabulated for use in LBL codes. This procedure was executed with the multi-spectral fitting routine using the fitted LBL parameters and abridged LM elements presented here. The resulting Y-factors are given in Table 22 .

To achieve consistent calibrations of the laboratory FTS data it was necessary to pass the near-infrared radiation through a sealed potassium cell and observe the $\mathrm{D}_{1}\left(12985.2 \mathrm{~cm}^{-1}\right)$ and $\mathrm{D}_{2}\left(13042.9 \mathrm{~cm}^{-1}\right)$ transitions in absorption. Unlike saturated-dip spectroscopy [35], the FTS resolution blends together the hyperfine and isotopic components of these features and a high fidelity retrieval of the convolved lineshape requires a database of line positions and intensities. Somewhat disparate sources of this information were identified, and an appropriate quantum model was developed to reproduce essential aspects of each. This was accomplished using first the SPFIT/SPCAT software [65] in which the atomic ground and excited states were defined as the ground rotational states of 'molecules' with exaggerated rotational constants. Over-determined fits provided effective spin-splitting parameters for these states which then enabled predictions of the relative intensities based on the $6 j$ coefficients that govern the relative intensities of the transitions. The total intensity is determined by the transition moment of $19.326 \times 10^{-30}$ Cm (5.7939 D) [66] and indicates that potassium partial pressures in the calibration cell are on the order of $3 \mathrm{mPa}(20 \mu \mathrm{Torr})$. SPCAT predictions for each of the three most abundant potassium isotopes were generated and then combined into a single HITRAN format listing in which the isotopic abundances [67] are utilized to adjust the relative intensities of the isotopic features.

Reference data 23 was compiled for input into the multispectrum fitting program and is based on positions from [35], the oscillator strength reported by Safranova [66] and the IUPAC isotope fractions [67]. It is presented in HITRAN format with MOL (molecule ID) of 41, ISO (isotope ID) of $1\left({ }^{39} \mathrm{~K}\right)$ and $2\left({ }^{41} \mathrm{~K}\right)$, units of $\mathrm{cm}^{-1}$ for line position (WAVE), units of $\mathrm{cm}^{-1} /\left(\right.$ molecule $\left.\mathrm{cm}^{2}\right)$ for line intensity (STREN), units of $\mathrm{s}^{-} 1$ for Einstein $A$ coefficients (ACOEFF), units of $\mathrm{cm}^{-1}$ for lower state energies (ELOW) and default values for all other parameters, quantum labels refer to (superfluous) isotope labels, upper state orbital angular momentum $\left(\mathbf{L}^{\prime}\right)$, upper state orbital angular momentum projection (parity), upper state orbital and spin angular momentum $\left(\mathbf{J}^{\prime}=\mathbf{L} \mathbf{~}^{\prime}+\mathbf{S}^{\prime}\right.$, where $\mathbf{S}$ is the electronic spin angular momentum), upper state total angular momentum, $\left(\mathbf{F}^{\prime}=\mathbf{J} '+\mathbf{I}^{\prime}\right.$, where 
Table 22: Y-factors determined at four temperatures from the best fit LBL parameters and augmented LM. Units are $\mathrm{cm}^{-1} \mathrm{~atm}^{-1}(0.29587215 \mathrm{MHz} / \mathrm{Pa})$

\begin{tabular}{|c|c|c|c|c|}
\hline quanta & $\mathrm{Y}(200 \mathrm{~K})$ & $\mathrm{Y}(250 \mathrm{~K})$ & $\mathrm{Y}(296 \mathrm{~K})$ & $\mathrm{Y}(340 \mathrm{~K})$ \\
\hline P35 P35 & 0.443827 & 0.036884 & 0.011486 & 0.00629 \\
\hline P34 Q34 & 0.348540 & 0.030827 & 0.010002 & 0.005625 \\
\hline P33 P33 & 0.149422 & 0.017622 & 0.006543 & 0.003894 \\
\hline P32 Q32 & 0.119395 & 0.015009 & 0.00579 & 0.003524 \\
\hline P31 P31 & 0.057017 & 0.009476 & 0.004168 & 0.002693 \\
\hline P30 Q30 & 0.046533 & 0.008245 & 0.003755 & 0.002472 \\
\hline P29 P29 & 0.024930 & 0.005718 & 0.002911 & 0.00201 \\
\hline P28 Q28 & 0.020859 & 0.005084 & 0.002665 & 0.001867 \\
\hline P27 P27 & 0.012586 & 0.003836 & 0.002193 & 0.00159 \\
\hline P26 Q26 & 0.010820 & 0.003476 & 0.002034 & 0.00149 \\
\hline P25 P25 & 0.007314 & 0.002810 & 0.001751 & 0.001312 \\
\hline P24 Q24 & 0.006453 & 0.002585 & 0.001638 & 0.001236 \\
\hline P23 P23 & 0.004810 & 0.002200 & 0.001455 & 0.001112 \\
\hline P22 Q22 & 0.004335 & 0.002045 & 0.001368 & 0.001051 \\
\hline P21 P21 & 0.003481 & 0.001802 & 0.001239 & 0.000956 \\
\hline P20 Q20 & 0.003186 & 0.001684 & 0.001166 & 0.000901 \\
\hline P19 P19 & 0.002689 & 0.001513 & 0.001064 & 0.000822 \\
\hline P18 Q18 & 0.002480 & 0.001414 & 0.000998 & 0.000771 \\
\hline P17 P17 & 0.002154 & 0.001279 & 0.000908 & 0.000696 \\
\hline P16 Q16 & 0.001988 & 0.001189 & 0.000844 & 0.000644 \\
\hline P15 P15 & 0.001742 & 0.001066 & 0.000755 & 0.000568 \\
\hline P14 Q14 & 0.001594 & 0.000976 & 0.000687 & 0.000512 \\
\hline P13 P13 & 0.001379 & 0.000850 & 0.00059 & 0.000427 \\
\hline P12 Q12 & 0.001231 & 0.000751 & 0.000511 & 0.00036 \\
\hline P11 P11 & 0.001015 & 0.000608 & 0.000396 & 0.00026 \\
\hline P10 Q10 & 0.000850 & 0.000489 & 0.000299 & 0.000176 \\
\hline P9 P9 & 0.000604 & 0.000312 & 0.000153 & $4.89 \mathrm{E}-05$ \\
\hline P8 Q8 & 0.000397 & 0.000155 & $2.2 \mathrm{E}-05$ & $-6.5 \mathrm{E}-05$ \\
\hline P7 P7 & $8.8 \mathrm{E}-05$ & $-7.9 \mathrm{E}-05$ & -0.00017 & -0.00023 \\
\hline P6 Q6 & -0.000210 & -0.00031 & -0.00037 & -0.0004 \\
\hline P5 P5 & -0.000630 & -0.00064 & -0.00064 & -0.00064 \\
\hline P4 Q4 & -0.001140 & -0.00104 & -0.00098 & -0.00093 \\
\hline P3 P3 & -0.001740 & -0.00151 & -0.00137 & -0.00126 \\
\hline P2 Q2 & -0.002980 & -0.00251 & -0.00221 & -0.00199 \\
\hline P1 P1 & -0.003410 & -0.00283 & -0.00246 & -0.0022 \\
\hline R1 R1 & 0.004643 & 0.003873 & 0.003364 & 0.002991 \\
\hline R2 Q2 & 0.004719 & 0.003913 & 0.003386 & 0.003002 \\
\hline R3 R3 & 0.003353 & 0.002879 & 0.002551 & 0.002302 \\
\hline R4 Q4 & 0.002639 & 0.002303 & 0.00206 & 0.001873 \\
\hline R5 R5 & 0.001960 & 0.001811 & 0.001681 & 0.001568 \\
\hline R6 Q6 & 0.001481 & 0.001427 & 0.001354 & 0.001283 \\
\hline R7 R7 & 0.000929 & 0.001043 & 0.001068 & 0.00106 \\
\hline R8 Q8 & 0.000575 & 0.000763 & 0.000831 & 0.000853 \\
\hline R9 R9 & $2.81 \mathrm{E}-05$ & 0.000400 & 0.000568 & 0.000655 \\
\hline R10 Q10 & -0.00027 & 0.000173 & 0.000378 & 0.00049 \\
\hline R11 R11 & -0.00088 & -0.00021 & 0.00011 & 0.000295 \\
\hline R12 Q12 & -0.00116 & -0.00042 & $-5.7 \mathrm{E}-05$ & 0.000151 \\
\hline R13 R13 & -0.00191 & -0.00086 & -0.00035 & $-5.7 \mathrm{E}-05$ \\
\hline R14 Q14 & -0.00220 & -0.00106 & -0.00051 & -0.00019 \\
\hline R15 R15 & -0.00320 & -0.00161 & -0.00086 & -0.00042 \\
\hline R16 Q16 & -0.00353 & -0.00183 & -0.00103 & -0.00057 \\
\hline $\mathrm{R} 17 \mathrm{R} 17$ & -0.00494 & -0.00252 & -0.00143 & -0.00081 \\
\hline R18 Q18 & -0.00539 & -0.0028 & -0.00164 & -0.00099 \\
\hline R19 R19 & -0.00747 & -0.00368 & -0.00207 & -0.00119 \\
\hline R20 Q20 & -0.00817 & -0.0041 & -0.0024 & -0.00146 \\
\hline R21 R21 & -0.01078 & -0.00458 & -0.00218 & -0.00093 \\
\hline R22 Q22 & -0.01263 & -0.00586 & -0.00325 & -0.00188 \\
\hline R23 R23 & -0.01809 & -0.00683 & -0.00295 & -0.00109 \\
\hline R24 Q24 & -0.01958 & -0.00743 & -0.00327 & -0.00123 \\
\hline $\mathrm{R} 25 \mathrm{R} 25$ & -0.03096 & -0.00843 & -0.00159 & 0.001456 \\
\hline R26 Q26 & -0.03597 & -0.01111 & -0.00369 & -0.00037 \\
\hline $\mathrm{R} 27 \mathrm{R} 27$ & -0.03414 & 0.017929 & 0.031994 & 0.037857 \\
\hline R28 Q28 & -0.05761 & $-9.5 \mathrm{E}-05$ & 0.014885 & 0.02101 \\
\hline R29 R29 & -0.47271 & -0.27087 & -0.21182 & -0.18302 \\
\hline R30 Q30 & -0.42595 & -0.22924 & -0.17607 & -0.15122 \\
\hline R31 R31 & -0.50903 & -0.11173 & -0.03878 & -0.01344 \\
\hline R32 Q32 & -0.50235 & -0.0863 & -0.0188 & 0.002795 \\
\hline R33 R33 & -1.53951 & -0.2404 & -0.06827 & -0.02096 \\
\hline R34 Q34 & -1.58388 & -0.21832 & -0.05157 & -0.00825 \\
\hline R35 R35 & -6.20458 & -0.64675 & -0.15059 & -0.0419 \\
\hline
\end{tabular}


Table 23: Potassium linelist. Units are $\mathrm{cm}^{-1}$ for $\sigma_{0}$ and $E " ; \mathrm{cm}^{-1} /\left(\right.$ molec $\left.\mathrm{cm}^{-2}\right)$ for $I(296$ $\mathrm{K})$, natural abundance, $a$ [unitless] is included for $I$. Quanta are described in the text, masses are in amu.

\begin{tabular}{|c|c|c|c|c|c|c|c|c|c|c|c|c|}
\hline$\sigma_{0}$ & $I(296 \mathrm{~K})$ & $E^{\prime \prime}$ & $L^{\prime}$ & $p^{\prime}$ & $J^{\prime}+\frac{1}{2}$ & $F^{\prime}$ & $L "$ & $p "$ & $J^{\prime \prime}+\frac{1}{2}$ & $F "$ & $\operatorname{mass}[68]$ & $a[67]$ \\
\hline 12985.178261 & $8.808 \mathrm{E}-15$ & 0.0150 & 1 & -1 & 1 & 1 & 0 & 0 & 1 & 2 & 38.96370668 & 0.932581 \\
\hline 12985.180114 & $8.808 \mathrm{E}-15$ & 0.0150 & 1 & -1 & 1 & 2 & 0 & 0 & 1 & 2 & 38.96370668 & 0.932581 \\
\hline 12985.193662 & $1.765 \mathrm{E}-15$ & 0.0000 & 1 & -1 & 1 & 1 & 0 & 0 & 1 & 1 & 38.96370668 & 0.932581 \\
\hline 12985.195515 & $8.828 \mathrm{E}-15$ & 0.0000 & 1 & -1 & 1 & 2 & 0 & 0 & 1 & 1 & 38.96370668 & 0.932581 \\
\hline 13042.889156 & $1.774 \mathrm{E}-15$ & 0.0150 & 1 & -1 & 2 & 1 & 0 & 0 & 1 & 2 & 38.96370668 & 0.932581 \\
\hline 13042.889531 & $8.848 \mathrm{E}-15$ & 0.0150 & 1 & -1 & 2 & 2 & 0 & 0 & 1 & 2 & 38.96370668 & 0.932581 \\
\hline 13042.890180 & $2.482 \mathrm{E}-14$ & 0.0150 & 1 & -1 & 2 & 3 & 0 & 0 & 1 & 2 & 38.96370668 & 0.932581 \\
\hline 13042.904493 & $3.547 \mathrm{E}-15$ & 0.0000 & 1 & -1 & 2 & 0 & 0 & 0 & 1 & 1 & 38.96370668 & 0.932581 \\
\hline 13042.904558 & $8.848 \mathrm{E}-15$ & 0.0000 & 1 & -1 & 2 & 1 & 0 & 0 & 1 & 1 & 38.96370668 & 0.932581 \\
\hline 13042.904932 & $8.848 \mathrm{E}-15$ & 0.0000 & 1 & -1 & 2 & 2 & 0 & 0 & 1 & 1 & 38.96370668 & 0.932581 \\
\hline 12985.189237 & $6.371 \mathrm{E}-16$ & 0.0080 & 1 & -1 & 1 & 1 & 0 & 0 & 1 & 2 & 40.96182576 & 0.067302 \\
\hline 12985.190254 & $6.371 \mathrm{E}-16$ & 0.0080 & 1 & -1 & 1 & 2 & 0 & 0 & 1 & 2 & 40.96182576 & 0.067302 \\
\hline 12985.197710 & $1.274 \mathrm{E}-16$ & 0.0000 & 1 & -1 & 1 & 1 & 0 & 0 & 1 & 1 & 40.96182576 & 0.067302 \\
\hline 12985.198727 & $6.371 \mathrm{E}-16$ & 0.0000 & 1 & -1 & 1 & 2 & 0 & 0 & 1 & 1 & 40.96182576 & 0.067302 \\
\hline 13042.899917 & $1.277 \mathrm{E}-16$ & 0.0080 & 1 & -1 & 2 & 1 & 0 & 0 & 1 & 2 & 40.96182576 & 0.067302 \\
\hline 13042.900029 & $6.386 \mathrm{E}-16$ & 0.0080 & 1 & -1 & 2 & 2 & 0 & 0 & 1 & 2 & 40.96182576 & 0.067302 \\
\hline 13042.900478 & $1.791 \mathrm{E}-15$ & 0.0080 & 1 & -1 & 2 & 3 & 0 & 0 & 1 & 2 & 40.96182576 & 0.067302 \\
\hline 13042.908389 & $2.560 \mathrm{E}-16$ & 0.0000 & 1 & -1 & 2 & 0 & 0 & 0 & 1 & 1 & 40.96182576 & 0.067302 \\
\hline 13042.908390 & $6.386 \mathrm{E}-16$ & 0.0000 & 1 & -1 & 2 & 1 & 0 & 0 & 1 & 1 & 40.96182576 & 0.067302 \\
\hline 13042.908502 & $6.386 \mathrm{E}-16$ & 0.0000 & 1 & -1 & 2 & 2 & 0 & 0 & 1 & 1 & 40.96182576 & 0.067302 \\
\hline
\end{tabular}

I is the potassium nuclear angular momentum), lower state orbital angular momentum (L"), lower state orbital angular momentum projection (parity), lower state orbital and spin angular momentum $(\mathbf{J} "=\mathbf{L} "+\mathbf{S} ")$ and the lower state total angular momentum $(\mathbf{F} "=\mathbf{J} "+\mathbf{I} ")$. 